\title{
Ricci curvature, entropy and optimal transport
}

\author{
Shin-ichi OHTA* \\ Department of Mathematics, Faculty of Science, Kyoto University, \\ Kyoto 606-8502, JAPAN (e-mail: sohta@math.kyoto-u.ac.jp)
}

\begin{abstract}
This is the lecture notes on the interplay between optimal transport and Riemannian geometry. On a Riemannian manifold, the convexity of entropy along optimal transport in the space of probability measures characterizes lower bounds of the Ricci curvature. We then discuss geometric properties of general metric measure spaces satisfying this convexity condition.
\end{abstract}

Mathematics Subject Classification (2000): 53C21, 53C23, 53C60, 28A33, $28 \mathrm{D} 20$

Keywords: Ricci curvature, entropy, optimal transport, curvature-dimension condition

\section{Introduction}

This article is extended notes based on the author's lecture series in summer school at Université Joseph Fourier, Grenoble: 'Optimal Transportation: Theory and Applications'. The aim of these five lectures (corresponding to Sections 3 17) was to review the recent impressive development on the interplay between optimal transport theory and Riemannian geometry. Ricci curvature and entropy are the key ingredients. See [Lo2] for a survey in the same spirit with a slightly different selection of topics.

Optimal transport theory is concerned with the behavior of transport between two probability measures in a metric space. We say that such transport is optimal if it minimizes a certain cost function typically defined from the distance of the metric space. Optimal transport naturally inherits the geometric structure of the underlying space, especially Ricci curvature plays a crucial role for describing optimal transport in Riemannian manifolds. In fact, optimal transport is always performed along geodesics, and we obtain Jacobi fields as their variational vector fields. The behavior of these Jacobi fields is controlled by the Ricci curvature as is usual in comparison geometry. In this way, a lower Ricci curvature bound turns out to be equivalent to a certain convexity property of entropy in the space of probability measures. The latter convexity condition is called the curvature-dimension condition, and it can be formulated without using the differentiable

*Partly supported by the Grant-in-Aid for Young Scientists (B) 20740036. 
structure. Therefore the curvature-dimension condition can be regarded as a 'definition' of a lower Ricci curvature bound for general metric measure spaces, and implies many analogous properties in an interesting way.

A prerequisite is the basic knowledge of optimal transport theory and Wasserstein geometry. Riemannian geometry is also necessary in Sections 3, 4, and is helpful for better understanding of the other sections. We refer to AGS], Vi1], Vi2 and other articles in this proceeding for optimal transport theory, [CE], $\mathrm{Ch}$ ] and [Sak] for the basics of (comparison) Riemannian geometry. We discuss Finsler geometry in Section 7 , for which we refer to [BCS], [Sh2] and [Oh5]. Besides them, main references are [CMS1, [CMS2], [vRS], [St3], [St4], [LV2], [LV1] and [Vi2, Chapter III].

The organization of this article is as follows. After summarizing some notations we use, Section 3 is devoted to the definition of the Ricci curvature of Riemannian manifolds and to the classical Bishop-Gromov volume comparison theorem. In Section 4, we start with the Brunn-Minkowski inequalities in (unweighted or weighted) Euclidean spaces, and explain the equivalence between a lower (weighted) Ricci curvature bound for a (weighted) Riemannian manifold and the curvature-dimension condition. In Section 5, we give the precise definition of the curvature-dimension condition for metric measure spaces, and see that it is stable under the measured Gromov-Hausdorff convergence. Section 6 is concerned with several geometric applications of the curvature-dimension condition followed by related open questions. In Section 7, we verify that this kind of machinery is useful also in Finsler geometry. We finally discuss three related topics in Section 8 , Interested readers can find more references in Further Reading at the end of each section (except the last section).

Some subjects in this article are more comprehensively discussed in [Vi2, Part III]. Despite these inevitable overlaps with Villani's massive book, we try to argue in a more geometric way, and mention recent development. Analytic applications of the curvaturedimension condition are not dealt with in these notes, for which we refer to [LV1], LV2] and [Vi2, Chapter III] among others.

I would like to express my gratitude to the organizers for the kind invitation to the fascinating summer school, and to all the audience for their attendance and interest. I also thank the referee for careful reading and valuable suggestions.

\section{Notations}

Throughout the article except Section $7,(M, g)$ is an $n$-dimensional, connected, complete $C^{\infty}$-Riemannian manifold without boundary such that $n \geq 2, \operatorname{vol}_{g}$ stands for the Riemannian volume measure of $g$. A weighted Riemannian manifold $(M, g, m)$ will mean a Riemannian manifold $(M, g)$ endowed with a conformal deformation $m=e^{-\psi} \operatorname{vol}_{g}$ of $\operatorname{vol}_{g}$ with $\psi \in C^{\infty}(M)$. Similarly, a weighted Euclidean space $\left(\mathbb{R}^{n},\|\cdot\|, m\right)$ will be a Euclidean space with a measure $m=e^{-\psi} \operatorname{vol}_{n}$, where $\operatorname{vol}_{n}$ stands for the $n$-dimensional Lebesgue measure.

A metric space is called a geodesic space if any two points $x, y \in X$ can be connected by a rectifiable curve $\gamma:[0,1] \longrightarrow X$ of length $d(x, y)$ with $\gamma(0)=x$ and $\gamma(1)=y$. Such minimizing curves parametrized proportionally to arc length are called minimal geodesics. 
The open ball of center $x$ and radius $r$ will be denoted by $B(x, r)$. We remark that, thanks to the Hopf-Rinow theorem (cf. [Bal, Theorem 2.4]), a complete, locally compact geodesic space is proper, i.e., every bounded closed set is compact.

In this article, we mean by a metric measure space a triple $(X, d, m)$ consisting of a complete, separable geodesic space $(X, d)$ and a Borel measure $m$ on it. Our definition of the curvature-dimension condition will include the additional (but natural) condition that $0<m(B(x, r))<\infty$ holds for all $x \in X$ and $0<r<\infty$. We extend $m$ to an outer measure in the Brunn-Minkowski inequalities (Theorems 4.1, 4.3, 6.1, see Remark 4.2 for more details).

For a complete, separable metric space $(X, d), \mathcal{P}(X)$ stands for the set of Borel probability measures on $X$. Define $\mathcal{P}_{2}(X) \subset \mathcal{P}(X)$ as the set of measures of finite second moment (i.e., $\int_{X} d(x, y)^{2} d \mu(y)<\infty$ for some (and hence all) $x \in X$ ). We denote by $\mathcal{P}_{b}(X) \subset \mathcal{P}_{2}(X), \mathcal{P}_{c}(X) \subset \mathcal{P}_{b}(X)$ the sets of measures of bounded or compact support, respectively. Given a measure $m$ on $X$, denote by $\mathcal{P}^{\text {ac }}(X, m) \subset \mathcal{P}(X)$ the set of absolutely continuous measures with respect to $m$. Then $d_{2}^{W}$ stands for the $L^{2}$-(KantorovichRubinstein-) Wasserstein distance of $\mathcal{P}_{2}(X)$. The push-forward of a measure $\mu$ by a map $\mathcal{F}$ will be written as $\mathcal{F}_{\sharp} \mu$.

As usual in comparison geometry, the following functions will frequently appear in our discussions. For $K \in \mathbb{R}, N \in(1, \infty)$ and $0<r(<\pi \sqrt{(N-1) / K}$ if $K>0)$, we set

$$
\mathbf{s}_{K, N}(r):=\left\{\begin{array}{cl}
\sqrt{(N-1) / K} \sin (r \sqrt{K /(N-1)}) & \text { if } K>0, \\
r & \text { if } K=0, \\
\sqrt{-(N-1) / K} \sinh (r \sqrt{-K /(N-1)}) & \text { if } K<0 .
\end{array}\right.
$$

This is the solution to the differential equation

$$
\mathbf{s}_{K, N}^{\prime \prime}+\frac{K}{N-1} \mathbf{s}_{K, N}=0
$$

with the initial conditions $\mathbf{s}_{K, N}(0)=0$ and $\mathbf{s}_{K, N}^{\prime}(0)=1$. For $n \in \mathbb{N}$ with $n \geq 2, \mathbf{s}_{K, n}(r)^{n-1}$ is proportional to the area of the sphere of radius $r$ in the $n$-dimensional space form of constant sectional curvature $K /(n-1)$ (see Theorem 3.2 and the paragraph after it). In addition, using $\mathbf{s}_{K, N}$, we define

$$
\beta_{K, N}^{t}(r):=\left(\frac{\mathbf{s}_{K, N}(t r)}{t \mathbf{s}_{K, N}(r)}\right)^{N-1}, \quad \beta_{K, \infty}^{t}(r):=e^{K\left(1-t^{2}\right) r^{2} / 6}
$$

for $K, N, r$ as above and $t \in(0,1)$. This function plays a vital role in the key infinitesimal inequality (4.14) of the curvature-dimension condition.

\section{Ricci curvature and comparison theorems}

We begin with the basic concepts of curvature in Riemannian geometry and several comparison theorems involving lower bounds of the Ricci curvature. Instead of giving the detailed definition, we intend to explain the geometric intuition of the sectional and Ricci curvatures through comparison geometry. 
Curvature is one of the most important quantities in Riemannian geometry. By putting some conditions on the value of the (sectional or Ricci) curvature, we obtain various quantitative and qualitative controls of distance, measure, geodesics and so forth. Comparison geometry is specifically interested in spaces whose curvature is bounded by a constant from above or below. In other words, we consider a space which is more positively or negatively curved than a space form of constant curvature, and compare these spaces from various viewpoints.

The $n$-dimensional (simply connected) space form $\mathbb{M}^{n}(k)$ of constant sectional curvature $k \in \mathbb{R}$ is the unit sphere $\mathbb{S}^{n}$ for $k=1$; the Euclidean space $\mathbb{R}^{n}$ for $k=0$; and the hyperbolic space $\mathbb{H}^{n}$ for $k=-1$. Scaling gives general space forms for all $k \in \mathbb{R}$, e.g., $\mathbb{M}^{n}(k)$ for $k>0$ is the sphere of radius $1 / \sqrt{k}$ in $\mathbb{R}^{n+1}$ with the induced Riemannian metric.

\subsection{Sectional curvature}

Given linearly independent tangent vectors $v, w \in T_{x} M$, the sectional curvature $\mathcal{K}(v, w) \in$ $\mathbb{R}$ reflects the asymptotic behavior of the distance function $d(\gamma(t), \eta(t))$ near $t=0$ between geodesics $\gamma(t)=\exp _{x}(t v)$ and $\eta(t)=\exp _{x}(t w)$. That is to say, the asymptotic behavior of $d(t):=d(\gamma(t), \eta(t))$ near $t=0$ is same as the distance between geodesics, with the same speed and angle between them, in the space form of curvature $k=\mathcal{K}(v, w)$. (See Figure 1 which represents isometric embeddings of $\gamma$ and $\eta$ into $\mathbb{R}^{2}$ such that $d(\gamma(t), \eta(t))$ coincides with the Euclidean distance.)

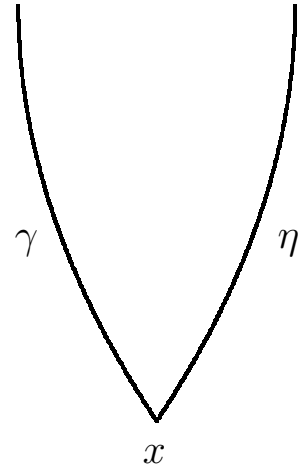

$\mathcal{K}>0$

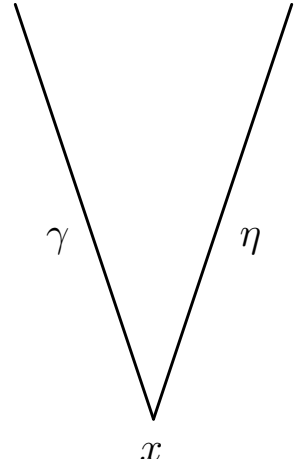

$\mathcal{K}=0$

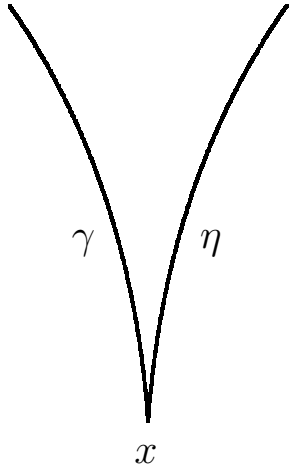

$\mathcal{K}<0$

Figure 1

Assuming $\|v\|=\|w\|=1$ for simplicity, we can compute $d(t)$ in the space form $\mathbb{M}^{n}(k)$ by using the spherical/Euclidean/hyperbolic law of cosines as (cf. [Sak, Section IV.1])

$$
\begin{aligned}
\cos (\sqrt{k} d(t)) & =\cos ^{2}(\sqrt{k} t)+\sin ^{2}(\sqrt{k} t) \cos \angle(v, w) & & \text { for } k>0 \\
d(t)^{2} & =2 t^{2}-2 t^{2} \cos \angle(v, w) & & \text { for } k=0 \\
\cosh (\sqrt{-k} d(t)) & =\cosh ^{2}(\sqrt{-k} t)-\sinh ^{2}(\sqrt{-k} t) \cos \angle(v, w) & & \text { for } k<0 .
\end{aligned}
$$


Observe that the dimension $n$ does not appear in these formulas. The sectional curvature $\mathcal{K}(v, w)$ depends only on the 2-plane (in $\left.T_{x} M\right)$ spanned by $v$ and $w$, and coincides with the Gaussian curvature at $x$ if $n=2$.

More precise relation between curvature and geodesics can be described through Jacobi fields. A $C^{\infty}$-vector field $J$ along a geodesic $\gamma:[0, l] \longrightarrow M$ is called a Jacobi field if it solves the Jacobi equation

$$
D_{\dot{\gamma}} D_{\dot{\gamma}} J(t)+R(J(t), \dot{\gamma}(t)) \dot{\gamma}(t)=0
$$

for all $t \in[0, l]$. Here $D_{\dot{\gamma}}$ denotes the covariant derivative along $\gamma$, and $R: T_{x} M \otimes$ $T_{x} M \longrightarrow T_{x}^{*} M \otimes T_{x} M$ is the curvature tensor determined by the Riemannian metric $g$. Another equivalent way of introducing a Jacobi field is to define it as the variational vector field $J(t)=(\partial \sigma / \partial s)(0, t)$ of some $C^{\infty}$-variation $\sigma:(-\varepsilon, \varepsilon) \times[0, l] \longrightarrow M$ such that $\sigma(0, t)=\gamma(t)$ and that every $\sigma_{s}:=\sigma(s, \cdot)$ is geodesic. (This characterization of Jacobi fields needs only the class of geodesics, and then it is possible to regard (3.1) as the definition of $R$.) For linearly independent vectors $v, w \in T_{x} M$, the precise definition of the sectional curvature is

$$
\mathcal{K}(v, w):=\frac{\langle R(w, v) v, w\rangle}{\|v\|^{2}\|w\|^{2}-\langle v, w\rangle^{2}} .
$$

It might be helpful to compare (3.1) with (2.2).

Remark 3.1 (Alexandrov spaces) Although it is not our main subject, we briefly comment on comparison geometry involving lower bounds of the sectional curvature. As the sectional curvature is defined for each two-dimensional subspace in tangent spaces, it controls the behavior of two-dimensional subsets in $M$, in particular, triangles. The classical Alexandrov-Toponogov comparison theorem asserts that $\mathcal{K} \geq k$ holds for some $k \in \mathbb{R}$ if and only if every geodesic triangle in $M$ is thicker than the triangle with the same side lengths in $\mathbb{M}^{2}(k)$. See Figure 2 for more details, where $M$ is of $\mathcal{K} \geq k$, and then $d(x, w) \geq d(\tilde{x}, \tilde{w})$ holds between geodesic triangles with the same side lengths $(d(x, y)=d(\tilde{x}, \tilde{y}), d(y, z)=d(\tilde{y}, \tilde{z}), d(z, x)=d(\tilde{z}, \tilde{x}))$ as well as $d(y, w)=d(\tilde{y}, \tilde{w})$.

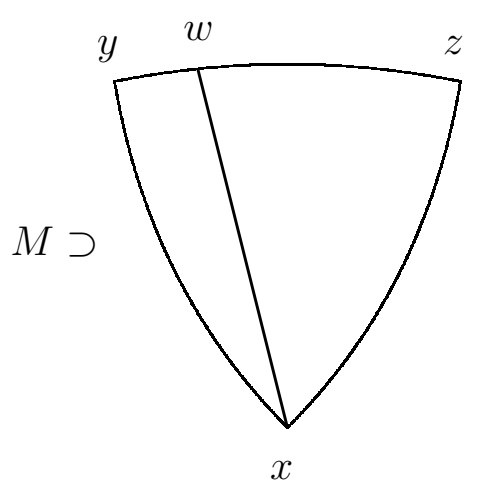

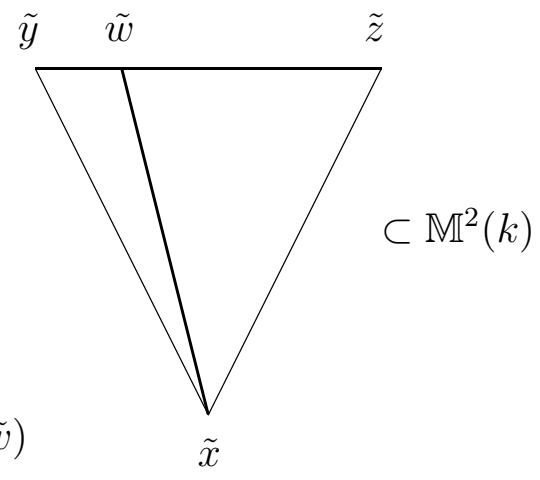

Figure 2 
The point is that we can forget about the dimension of $M$, because the sectional curvature cares only two-dimensional subsets. The above triangle comparison property is written by using only distance and geodesics, so that it can be formulated for metric spaces having enough geodesics (i.e., geodesic spaces). Such spaces are called Alexandrov spaces, and there are deep geometric and analytic theories on them (see [BGP], $\mathrm{OtS}],[\mathrm{BBI}$, Chapters 4,10]). We discuss optimal transport and Wasserstein geometry on Alexandrov spaces in Subsection 8.2 .

\subsection{Ricci curvature}

Given a unit vector $v \in T_{x} M$, we define the Ricci curvature of $v$ as the trace of the sectional curvature $\mathcal{K}(v, \cdot)$,

$$
\operatorname{Ric}(v):=\sum_{i=1}^{n-1} \mathcal{K}\left(v, e_{i}\right),
$$

where $\left\{e_{i}\right\}_{i=1}^{n-1} \cup\{v\}$ is an orthonormal basis of $T_{x} M$. We will mean by Ric $\geq K$ for $K \in \mathbb{R}$ that $\operatorname{Ric}(v) \geq K$ holds for all unit vectors $v \in T M$. As we discussed in the previous subsection, sectional curvature controls geodesics and distance. Ricci curvature has less information since we take the trace, and naturally controls the behavior of the measure $\operatorname{vol}_{g}$.

The following is one of the most important theorems in comparison Riemannian geometry, that asserts that a lower bound of the Ricci curvature implies an upper bound of the volume growth. The proof is done via calculations involving Jacobi fields. Recall (2.1) for the definition of the function $\mathbf{s}_{K, n}$.

Theorem 3.2 (Bishop-Gromov volume comparison) Assume that Ric $\geq K$ holds for some $K \in \mathbb{R}$. Then we have, for any $x \in M$ and $0<r<R(\leq \pi \sqrt{(n-1) / K}$ if $K>0)$,

$$
\frac{\operatorname{vol}_{g}(B(x, R))}{\operatorname{vol}_{g}(B(x, r))} \leq \frac{\int_{0}^{R} \mathbf{s}_{K, n}(t)^{n-1} d t}{\int_{0}^{r} \mathbf{s}_{K, n}(t)^{n-1} d t} .
$$

Proof. Given a unit vector $v \in T_{x} M$, we fix a unit speed minimal geodesic $\gamma:[0, l] \longrightarrow M$ with $\dot{\gamma}(0)=v$ and an orthonormal basis $\left\{e_{i}\right\}_{i=1}^{n-1} \cup\{v\}$ of $T_{x} M$. Then we consider the variation $\sigma_{i}:(-\varepsilon, \varepsilon) \times[0, l] \longrightarrow M$ defined by $\sigma_{i}(s, t):=\exp _{x}\left(t v+s t e_{i}\right)$ for $i=1, \ldots, n-1$, and introduce the Jacobi fields $\left\{J_{i}\right\}_{i=1}^{n-1}$ along $\gamma$ given by

$$
J_{i}(t):=\frac{\partial \sigma_{i}}{\partial s}(0, t)=D\left(\exp _{x}\right)_{t v}\left(t e_{i}\right) \in T_{\gamma(t)} M
$$

(see Figure 3, where $s>0$ ). 


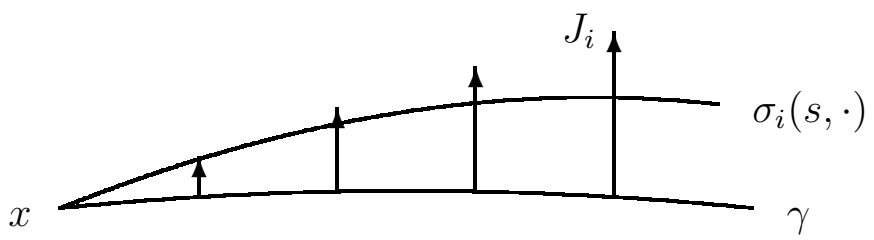

Figure 3

Note that $J_{i}(0)=0, D_{\dot{\gamma}} J_{i}(0)=e_{i},\left\langle J_{i}, \dot{\gamma}\right\rangle \equiv 0$ (by the Gauss lemma) and $\left\langle D_{\dot{\gamma}} J_{i}, \dot{\gamma}\right\rangle \equiv 0$ (by (3.1) and $\left.\left\langle R\left(J_{i}, \dot{\gamma}\right) \dot{\gamma}, \dot{\gamma}\right\rangle \equiv 0\right)$. We also remark that $\gamma(t)$ is not conjugate to $x$ for all $t \in(0, l)$ (and hence $\left\{J_{i}(t)\right\}_{i=1}^{n-1} \cup\{\dot{\gamma}(t)\}$ is a basis of $\left.T_{\gamma(t)} M\right)$ since $\gamma$ is minimal. Hence we find an $(n-1) \times(n-1)$ matrix $\mathcal{U}(t)=\left(u_{i j}(t)\right)_{i, j=1}^{n-1}$ such that $D_{\dot{\gamma}} J_{i}(t)=\sum_{j=1}^{n-1} u_{i j}(t) J_{j}(t)$ for $t \in(0, l)$. We define two more $(n-1) \times(n-1)$ matrices

$$
\mathcal{A}(t):=\left(\left\langle J_{i}(t), J_{j}(t)\right\rangle\right)_{i, j=1}^{n-1}, \quad \mathcal{R}(t):=\left(\left\langle R\left(J_{i}(t), \dot{\gamma}(t)\right) \dot{\gamma}(t), J_{j}(t)\right\rangle\right)_{i, j=1}^{n-1} .
$$

Note that $\mathcal{A}$ and $\mathcal{R}$ are symmetric matrices. Moreover, we have $\operatorname{tr}\left(\mathcal{R}(t) \mathcal{A}(t)^{-1}\right)=$ $\operatorname{Ric}(\dot{\gamma}(t))$ as $\mathcal{A}(t)$ is the matrix representation of the metric $g$ in the basis $\left\{J_{i}(t)\right\}_{i=1}^{n-1}$ of the orthogonal complement $\dot{\gamma}(t)^{\perp}$ of $\dot{\gamma}(t)$. To be precise, choosing an $(n-1) \times(n-1)$ matrix $\mathcal{C}=\left(c_{i j}\right)_{i, j=1}^{n-1}$ such that $\left\{\sum_{j=1}^{n-1} c_{i j} J_{j}(t)\right\}_{i=1}^{n-1}$ is orthonormal, we observe $I_{n}=\mathcal{C} \mathcal{A C}^{t}$ $\left(\mathcal{C}^{t}\right.$ is the transpose of $\mathcal{C}$ ) and

$$
\begin{aligned}
\operatorname{Ric}(\dot{\gamma}(t)) & =\sum_{i, j, k=1}^{n-1}\left\langle R\left(c_{i j} J_{j}(t), \dot{\gamma}(t)\right) \dot{\gamma}(t), c_{i k} J_{k}(t)\right\rangle=\operatorname{tr}\left(\mathcal{C}(t) \mathcal{R}(t) \mathcal{C}(t)^{t}\right) \\
& =\operatorname{tr}\left(\mathcal{R}(t) \mathcal{A}(t)^{-1}\right)
\end{aligned}
$$

Claim 3.3 (a) It holds that $\mathcal{U} \mathcal{A}=\mathcal{A U}^{t}$. In particular, we have $2 \mathcal{U}=\mathcal{A}^{\prime} \mathcal{A}^{-1}$.

(b) The matrix $\mathcal{U}$ is symmetric and we have $\operatorname{tr}\left(\mathcal{U}^{2}\right) \geq(\operatorname{tr} \mathcal{U})^{2} /(n-1)$.

Proof. (a) The first assertion easily follows from the Jacobi equation (3.1) and the symmetry of $\mathcal{R}$, indeed,

$$
\begin{aligned}
\frac{d}{d t}\left\{\left\langle D_{\dot{\gamma}} J_{i}, J_{j}\right\rangle-\left\langle J_{i}, D_{\dot{\gamma}} J_{j}\right\rangle\right\} & =\left\langle D_{\dot{\gamma}} D_{\dot{\gamma}} J_{i}, J_{j}\right\rangle-\left\langle J_{i}, D_{\dot{\gamma}} D_{\dot{\gamma}} J_{j}\right\rangle \\
& =-\left\langle R\left(J_{i}, \dot{\gamma}\right) \dot{\gamma}, J_{j}\right\rangle+\left\langle J_{i}, R\left(J_{j}, \dot{\gamma}\right) \dot{\gamma}\right\rangle=0 .
\end{aligned}
$$

Thus we have $\mathcal{A}^{\prime}=\mathcal{U} \mathcal{A}+\mathcal{A U}^{t}=2 \mathcal{U} \mathcal{A}$ which shows the second assertion.

(b) Recall that

$$
\frac{\partial \sigma_{i}}{\partial t}(0, t)=\dot{\gamma}(t), \quad \frac{\partial \sigma_{i}}{\partial s}(0, t)=J_{i}(t)
$$

hold for $t \in(0, l)$. As $[\partial / \partial s, \partial / \partial t]=0$, we have

$$
D_{\dot{\gamma}} J_{i}(t)=D_{t}\left(\frac{\partial \sigma_{i}}{\partial s}\right)(0, t)=D_{s}\left(\frac{\partial \sigma_{i}}{\partial t}\right)(0, t) .
$$


Now, we introduce the function

$$
f: \exp _{x}\left(\left\{t v+\sum_{i=1}^{n-1} s_{i} t e_{i}|t \in[0, l],| s_{i} \mid<\varepsilon\right\}\right) \rightarrow \mathbb{R}
$$

so that $f\left(\exp _{x}\left(t v+\sum_{i=1}^{n-1} s_{i} t e_{i}\right)\right)=t$. We derive from $\nabla f\left(\sigma_{i}(s, t)\right)=\left(\partial \sigma_{i} / \partial t\right)(s, t)$ that

$$
D_{s}\left(\frac{\partial \sigma_{i}}{\partial t}\right)(0, t)=D_{J_{i}}(\nabla f)(\gamma(t))=\nabla^{2} f\left(J_{i}(t)\right)
$$

where $\left\langle\nabla^{2} f(w), w^{\prime}\right\rangle=\operatorname{Hess} f\left(w, w^{\prime}\right)$. This means that $\mathcal{U}$ is the matrix presentation of the symmetric form $\nabla^{2} f$ (restricted in $\dot{\gamma}^{\perp}$ ) with respect to the basis $\left\{J_{i}\right\}_{i=1}^{n-1}$. Therefore $\mathcal{U}$ is symmetric. By denoting the eigenvalues of $\mathcal{U}$ by $\lambda_{1}, \ldots, \lambda_{n-1}$, the Cauchy-Schwarz inequality shows that

$$
(\operatorname{tr} \mathcal{U})^{2}=\left(\sum_{i=1}^{n-1} \lambda_{i}\right)^{2} \leq(n-1) \sum_{i=1}^{n-1} \lambda_{i}^{2}=(n-1) \operatorname{tr}\left(\mathcal{U}^{2}\right)
$$

We calculate, by using Claim 3.3(a),

$$
\begin{aligned}
{\left[(\operatorname{det} \mathcal{A})^{1 / 2(n-1)}\right]^{\prime} } & =\frac{1}{2(n-1)}(\operatorname{det} \mathcal{A})^{1 / 2(n-1)-1} \cdot \operatorname{det} \mathcal{A} \operatorname{tr}\left(\mathcal{A}^{\prime} \mathcal{A}^{-1}\right) \\
& =\frac{1}{n-1}(\operatorname{det} \mathcal{A})^{1 / 2(n-1)} \operatorname{tr} \mathcal{U}
\end{aligned}
$$

Then Claim $\underline{3.3}$ (b) yields

$$
\begin{aligned}
{\left[(\operatorname{det} \mathcal{A})^{1 / 2(n-1)}\right]^{\prime \prime} } & =\frac{1}{(n-1)^{2}}(\operatorname{det} \mathcal{A})^{1 / 2(n-1)}(\operatorname{tr} \mathcal{U})^{2}+\frac{1}{n-1}(\operatorname{det} \mathcal{A})^{1 / 2(n-1)} \operatorname{tr}\left(\mathcal{U}^{\prime}\right) \\
& \leq \frac{1}{n-1}(\operatorname{det} \mathcal{A})^{1 / 2(n-1)}\left\{\operatorname{tr}\left(\mathcal{U}^{2}\right)+\operatorname{tr}\left(\mathcal{U}^{\prime}\right)\right\}
\end{aligned}
$$

We also deduce from Claim 3.3(a) and (3.1) that

$$
\mathcal{U}^{\prime}=\frac{1}{2} \mathcal{A}^{\prime \prime} \mathcal{A}^{-1}-\frac{1}{2}\left(\mathcal{A}^{\prime} \mathcal{A}^{-1}\right)^{2}=\frac{1}{2}(-2 \mathcal{R}+2 \mathcal{U} \mathcal{A} \mathcal{U}) \mathcal{A}^{-1}-2 \mathcal{U}^{2}=-\mathcal{R} \mathcal{A}^{-1}-\mathcal{U}^{2} .
$$

This implies the (matrix) Riccati equation

$$
\mathcal{U}^{\prime}+\mathcal{U}^{2}+\mathcal{R} \mathcal{A}^{-1}=0
$$

Taking the trace gives

$$
(\operatorname{tr} \mathcal{U})^{\prime}+\operatorname{tr}\left(\mathcal{U}^{2}\right)+\operatorname{Ric}(\dot{\gamma})=0 .
$$

Thus we obtain from our hypothesis Ric $\geq K$ the differential inequality

$$
\left[(\operatorname{det} \mathcal{A})^{1 / 2(n-1)}\right]^{\prime \prime} \leq-\frac{K}{n-1}(\operatorname{det} \mathcal{A})^{1 / 2(n-1)} .
$$


This is a version of the fundamental Bishop comparison theorem which plays a prominent role in comparison geometry. Comparing (3.3) with (2.2), we have

$$
\begin{aligned}
& \frac{d}{d t}\left\{\left[(\operatorname{det} \mathcal{A})^{1 / 2(n-1)}\right]^{\prime} \mathbf{s}_{K, n}-(\operatorname{det} \mathcal{A})^{1 / 2(n-1)} \mathbf{s}_{K, n}^{\prime}\right\} \\
& =\left[(\operatorname{det} \mathcal{A})^{1 / 2(n-1)}\right]^{\prime \prime} \mathbf{s}_{K, n}-(\operatorname{det} \mathcal{A})^{1 / 2(n-1)} \mathbf{s}_{K, n}^{\prime \prime} \leq 0,
\end{aligned}
$$

and hence $(\operatorname{det} \mathcal{A})^{1 / 2(n-1)} / \mathbf{s}_{K, n}$ is non-increasing. Then integrating $\sqrt{\operatorname{det} \mathcal{A}}$ in unit vectors $v \in T_{x} M$ implies the area comparison theorem

$$
\frac{\operatorname{area}_{g}(S(x, R))}{\operatorname{area}_{g}(S(x, r))} \leq \frac{\mathbf{s}_{K, n}(R)^{n-1}}{\mathbf{s}_{K, n}(r)^{n-1}},
$$

where $S(x, r):=\{y \in M \mid d(x, y)=r\}$ and area ${ }_{g}$ stands for the $(n-1)$-dimensional Hausdorff measure associated with $g$ (in other words, the volume measure of the $(n-1)$ dimensional Riemannian metric of $S(x, r)$ induced from $g)$.

Now, we integrate (3.4) in the radial direction. Set $\mathbf{A}(t):=\operatorname{area}_{g}(S(x, t))$ and $\mathbf{S}(t):=$ $\mathbf{s}_{K, n}(t)^{n-1}$, and recall that $\mathbf{A} / \mathbf{S}$ is non-increasing. Hence we obtain the key inequality

$$
\int_{0}^{r} \mathbf{A} d t \int_{r}^{R} \mathbf{S} d t \geq \frac{\mathbf{A}(r)}{\mathbf{S}(r)} \int_{0}^{r} \mathbf{S} d t \int_{r}^{R} \mathbf{S} d t \geq \int_{0}^{r} \mathbf{S} d t \int_{r}^{R} \mathbf{A} d t .
$$

From here to the desired estimate (3.2) is the easy calculation as follows

$$
\begin{aligned}
& \operatorname{vol}_{g}(B(x, r)) \int_{0}^{R} \mathbf{s}_{K, n}(t)^{n-1} d t=\int_{0}^{r} \mathbf{A} d t \int_{r}^{R} \mathbf{S} d t+\int_{0}^{r} \mathbf{A} d t \int_{0}^{r} \mathbf{S} d t \\
& \geq \int_{0}^{r} \mathbf{S} d t \int_{r}^{R} \mathbf{A} d t+\int_{0}^{r} \mathbf{A} d t \int_{0}^{r} \mathbf{S} d t=\operatorname{vol}_{g}(B(x, R)) \int_{0}^{r} \mathbf{s}_{K, n}(t)^{n-1} d t .
\end{aligned}
$$

The sphere of radius $r$ in the space form $\mathbb{M}^{n}(k)$ has area $a_{n} \mathbf{s}_{(n-1) k, n}(r)^{n-1}$, where $a_{n}$ is the area of $\mathbb{S}^{n-1}$, and the ball of radius $r$ has volume $a_{n} \int_{0}^{r} \mathbf{s}_{(n-1) k, n}(t)^{n-1} d t$. Thus the right-hand side of (3.2) ((3.4), respectively) coincides with the ratio of the volume of balls (the area of spheres, respectively) of radius $R$ and $r$ in $\mathbb{M}^{n}(K /(n-1)$ ).

Theorem 3.2 for $K>0$ immediately implies a diameter bound. This ensures that the condition $R \leq \pi \sqrt{(n-1) / K}$ in Theorem 3.2 is natural.

Corollary 3.4 (Bonnet-Myers diameter bound) If Ric $\geq K>0$, then we have

$$
\operatorname{diam} M \leq \pi \sqrt{\frac{n-1}{K}}
$$

Proof. Put $R:=\pi \sqrt{(n-1) / K}$ and assume diam $M \geq R$. Given $x \in M$, Theorem 3.2 implies that

$$
\begin{aligned}
\limsup _{\varepsilon \downarrow 0} \frac{\operatorname{vol}_{g}(B(x, R) \backslash B(x, R-\varepsilon))}{\operatorname{vol}_{g}(B(x, R))} & =\limsup _{\varepsilon \downarrow 0}\left\{1-\frac{\operatorname{vol}_{g}(B(x, R-\varepsilon))}{\operatorname{vol}_{g}(B(x, R))}\right\} \\
& \leq \limsup _{\varepsilon \downarrow 0} \frac{\int_{R-\varepsilon}^{R} \mathbf{s}_{K, n}(t)^{n-1} d t}{\int_{0}^{R} \mathbf{s}_{K, n}(t)^{n-1} d t}=0 .
\end{aligned}
$$


This shows $\operatorname{area}_{g}(S(x, R))=0$ and hence diam $M \leq R$. To be precise, it follows from area $_{g}(S(x, R))=0$ that every point in $S(x, R)$ must be a conjugate point of $x$. Therefore any geodesic emanating from $x$ is not minimal after passing through $S(x, R)$, and hence $\operatorname{diam} M=R$. (A more direct proof in terms of metric geometry can be found in Theorem 6.5(i).)

The bound (3.6) is sharp, and equality is achieved only by the sphere in $\mathbb{M}^{n}(K /(n-1))$ of radius $\sqrt{(n-1) / K}$ (compare this with Theorem 6.6).

As we mentioned in Remark 3.1, lower sectional curvature bounds are characterized by simple triangle comparison properties involving only distance, and there is a successful theory of metric spaces satisfying them. Then it is natural to ask the following question.

Question 3.5 How to characterize lower Ricci curvature bounds without using differentiable structure?

This had been a long standing important question, and we will see an answer in the next section (Theorem 4.6). Such a condition naturally involves measure and dimension besides distance, and should be preserved under the convergence of metric measure spaces (see Section [5).

Further Reading See, for instances, $\mathrm{CE}$, $\mathrm{Ch}$ ] and $[\mathrm{Sak}$ for the fundamentals of Riemannian geometry and comparison theorems. A property corresponding to the Bishop comparison theorem (3.3) was proposed as a lower Ricci curvature bound for metric measure spaces by Cheeger and Colding [CC] (as well as Gromov [Gr]), and used to study the limit spaces of Riemannian manifolds with uniform lower Ricci curvature bounds. The deep theory of such limit spaces is one of the main motivations for asking Question 3.5, so that the stability deserves a particular interest (see Section 5 for more details). The systematic investigation of (3.3) in metric measure spaces has not been done until [Oh1] and St4] where we call this property the measure contraction property. We will revisit this in Subsection 8.3. Here we only remark that the measure contraction property is strictly weaker than the curvature-dimension condition.

\section{A characterization of lower Ricci curvature bound via optimal transport}

The Bishop-Gromov volume comparison theorem (Theorem 3.2) can be regarded as a concavity estimate of $\operatorname{vol}_{g}^{1 / n}$ along the contraction of the ball $B(x, R)$ to its center $x$. This is generalized to optimal transport between pairs of uniform distributions (the Brunn-Minkowski inequality) and, moreover, pairs of probability measures (the curvaturedimension condition). Figure 4 represents the difference between contraction and transport (see also Figures 5, 8). 


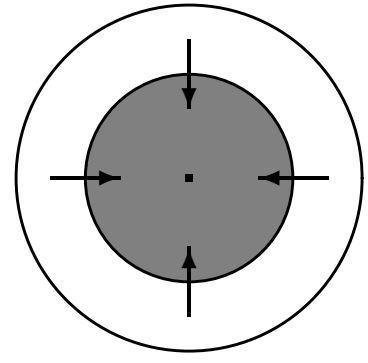

Bishop-Gromov

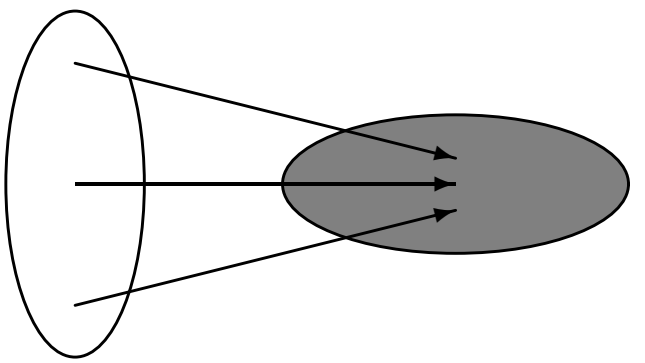

Brunn-Minkowski/Curvature-dimension

Figure 4

The main theorem in this section is Theorem 4.6 which asserts that, on a weighted Riemannian manifold, the curvature-dimension condition is equivalent to a lower bound of the corresponding weighted Ricci curvature. In order to avoid lengthy calculations, we begin with Euclidean spaces with or without weight, and see the relation between the Brunn-Minkowski inequality and the weighted Ricci curvature. Then the general Riemannian situation is only briefly explained. We hope that our simplified argument will help the readers to catch the idea of the curvature-dimension condition.

\subsection{Brunn-Minkowski inequalities in Euclidean spaces}

For later convenience, we explain fundamental facts of optimal transport theory on Euclidean spaces. Given $\mu_{0}, \mu_{1} \in \mathcal{P}_{c}\left(\mathbb{R}^{n}\right)$ with $\mu_{0} \in \mathcal{P}^{\text {ac }}\left(\mathbb{R}^{n}, \operatorname{vol}_{n}\right)$, there is a convex function $f: \mathbb{R}^{n} \longrightarrow \mathbb{R}$ such that the map

$$
\mathcal{F}_{t}(x):=(1-t) x+t \nabla f(x), \quad t \in[0,1],
$$

gives the unique optimal transport from $\mu_{0}$ to $\mu_{1}$ (Brenier's theorem, $\left.[\mathrm{Br}]\right)$. Precisely, $t \longmapsto \mu_{t}:=\left(\mathcal{F}_{t}\right)_{\sharp} \mu_{0}$ is the unique minimal geodesic from $\mu_{0}$ to $\mu_{1}$ with respect to the $L^{2}$-Wasserstein distance. We remark that the convex function $f$ is twice differentiable a.e. (Alexandrov's theorem, cf. [Vi2, Chapter 14]). Thus $\nabla f$ makes sense and $\mathcal{F}_{t}$ is differentiable a.e. Moreover, the Monge-Ampère equation

$$
\rho_{1}\left(\mathcal{F}_{1}(x)\right) \operatorname{det}\left(D \mathcal{F}_{1}(x)\right)=\rho_{0}(x)
$$

holds for $\mu_{0}$-a.e. $x$.

Now, we are ready for proving the classical Brunn-Minkowski inequality in the (unweighted) Euclidean space $\left(\mathbb{R}^{n},\|\cdot\|, \operatorname{vol}_{n}\right)$. Briefly speaking, it asserts that $\operatorname{vol}_{n}^{1 / n}$ is concave. We shall give a proof based on optimal transport theory. Given two (nonempty) sets $A, B \subset \mathbb{R}^{n}$ and $t \in[0,1]$, we set

$$
(1-t) A+t B:=\{(1-t) x+t y \mid x \in A, y \in B\} .
$$

(See Figure 5, where $(1 / 2) A+(1 / 2) B$ has much more measure than $A$ and $B$.) 


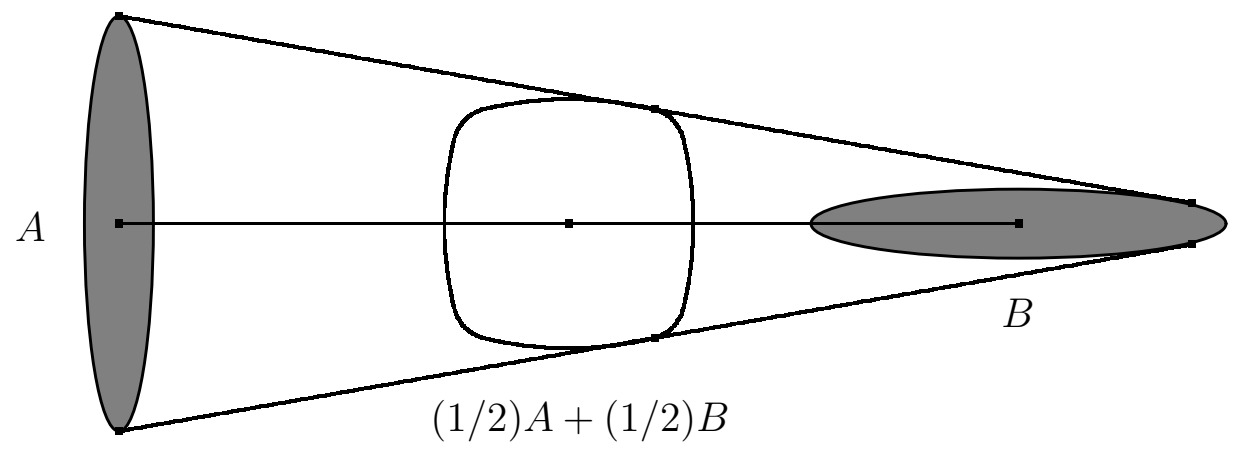

Figure 5

Theorem 4.1 (Brunn-Minkowski inequality) For any measurable sets $A, B \subset \mathbb{R}^{n}$ and $t \in[0,1]$, we have

$$
\operatorname{vol}_{n}((1-t) A+t B)^{1 / n} \geq(1-t) \operatorname{vol}_{n}(A)^{1 / n}+t \operatorname{vol}_{n}(B)^{1 / n} .
$$

Proof. We can assume that both $A$ and $B$ are bounded and of positive measure. The case of $\operatorname{vol}_{n}(A)=0$ is easily checked by choosing a point $x \in A$, as we have

$$
\operatorname{vol}_{n}((1-t) A+t B)^{1 / n} \geq \operatorname{vol}_{n}((1-t)\{x\}+t B)^{1 / n}=t \operatorname{vol}_{n}(B)^{1 / n} .
$$

If either $A$ or $B$ is unbounded, then applying (4.2) to bounded sets yields

$$
\begin{aligned}
& \operatorname{vol}_{n}((1-t)\{A \cap B(0, R)\}+t\{B \cap B(0, R)\})^{1 / n} \\
& \geq(1-t) \operatorname{vol}_{n}(A \cap B(0, R))^{1 / n}+t \operatorname{vol}_{n}(B \cap B(0, R))^{1 / n} .
\end{aligned}
$$

We take the limit as $R$ go to infinity and obtain

$$
\operatorname{vol}_{n}((1-t) A+t B)^{1 / n} \geq(1-t) \operatorname{vol}_{n}(A)^{1 / n}+t \operatorname{vol}_{n}(B)^{1 / n} .
$$

Consider the uniform distributions on $A$ and $B$,

$$
\mu_{0}=\rho_{0} \operatorname{vol}_{n}:=\frac{\chi_{A}}{\operatorname{vol}_{n}(A)} \operatorname{vol}_{n}, \quad \mu_{1}=\rho_{1} \operatorname{vol}_{n}:=\frac{\chi_{B}}{\operatorname{vol}_{n}(B)} \operatorname{vol}_{n},
$$

where $\chi_{A}$ stands for the characteristic function of $A$. As $\mu_{0}$ is absolutely continuous, there is a convex function $f: \mathbb{R}^{n} \longrightarrow \mathbb{R}$ such that the map $\mathcal{F}_{1}=(1-t) \operatorname{Id}_{\mathbb{R}^{n}}+t \nabla f, t \in[0,1]$, is the unique optimal transport from $\mu_{0}$ to $\mu_{1}$. Between the uniform distributions $\mu_{0}$ and $\mu_{1}$, the Monge-Ampère equation (4.1) simply means that

$$
\operatorname{det}\left(D \mathcal{F}_{1}\right)=\frac{\operatorname{vol}_{n}(B)}{\operatorname{vol}_{n}(A)}
$$

$\mu_{0}$-a.e.

Note that $D \mathcal{F}_{1}=$ Hess $f$ is symmetric and positive definite $\mu_{0}$-a.e., since $f$ is convex and $\operatorname{det}\left(D \mathcal{F}_{1}\right)>0$. We shall estimate $\operatorname{det}\left(D \mathcal{F}_{t}\right)=\operatorname{det}\left((1-t) I_{n}+t D \mathcal{F}_{1}\right)$ from above 
and below. To do so, we denote the eigenvalues of $D \mathcal{F}_{1}$ by $\lambda_{1}, \ldots, \lambda_{n}>0$ and apply the inequality of arithmetic and geometric means to see

$$
\begin{aligned}
& \left\{\frac{(1-t)^{n}}{\operatorname{det}\left((1-t) I_{n}+t D \mathcal{F}_{1}\right)}\right\}^{1 / n}+\left\{\frac{t^{n} \operatorname{det}\left(D \mathcal{F}_{1}\right)}{\operatorname{det}\left((1-t) I_{n}+t D \mathcal{F}_{1}\right)}\right\}^{1 / n} \\
& =\left\{\prod_{i=1}^{n} \frac{1-t}{(1-t)+t \lambda_{i}}\right\}^{1 / n}+\left\{\prod_{i=1}^{n} \frac{t \lambda_{i}}{(1-t)+t \lambda_{i}}\right\}^{1 / n} \\
& \leq \frac{1}{n} \sum_{i=1}^{n}\left\{\frac{1-t}{(1-t)+t \lambda_{i}}+\frac{t \lambda_{i}}{(1-t)+t \lambda_{i}}\right\}=1 .
\end{aligned}
$$

Thus we have, on the one hand,

$$
\operatorname{det}\left(D \mathcal{F}_{t}\right)^{1 / n} \geq(1-t)+t \operatorname{det}\left(D \mathcal{F}_{1}\right)^{1 / n}=(1-t)+t\left\{\frac{\operatorname{vol}_{n}(B)}{\operatorname{vol}_{n}(A)}\right\}^{1 / n}
$$

On the other hand, the Hölder inequality and the change of variables formula yield

$$
\int_{A} \operatorname{det}\left(D \mathcal{F}_{t}\right)^{1 / n} d \mu_{0} \leq\left(\int_{A} \operatorname{det}\left(D \mathcal{F}_{t}\right) d \mu_{0}\right)^{1 / n}=\left(\frac{1}{\operatorname{vol}_{n}(A)} \int_{\mathcal{F}_{t}(A)} d \operatorname{vol}_{n}\right)^{1 / n} .
$$

Therefore we obtain

$$
\int_{A} \operatorname{det}\left(D \mathcal{F}_{t}\right)^{1 / n} d \mu_{0} \leq\left\{\frac{\operatorname{vol}_{n}\left(\mathcal{F}_{t}(A)\right)}{\operatorname{vol}_{n}(A)}\right\}^{1 / n} \leq\left\{\frac{\operatorname{vol}_{n}((1-t) A+t B)}{\operatorname{vol}_{n}(A)}\right\}^{1 / n} .
$$

Combining these, we complete the proof of (4.2).

Remark 4.2 We remark that the set $(1-t) A+t B$ is not necessarily measurable (regardless the measurability of $A$ and $B)$. Hence, to be precise, $\operatorname{vol}_{n}((1-t) A+t B)$ is considered as an outer measure given by $\inf _{W} \operatorname{vol}_{n}(W)$, where $W \subset \mathbb{R}^{n}$ runs over all measurable sets containing $(1-t) A+t B$. The same remark is applied to Theorems 4.3 , 6.1 below.

Next we treat the weighted case $\left(\mathbb{R}^{n},\|\cdot\|, m\right)$, where $m=e^{-\psi} \operatorname{vol}_{n}$ with $\psi \in C^{\infty}\left(\mathbb{R}^{n}\right)$. Then we need to replace $1 / n$ in (4.2) with $1 / N$ for some $N \in(n, \infty)$, and the analogue of (4.2) leads us to an important condition on $\psi$.

Theorem 4.3 (Brunn-Minkowski inequality with weight) Take $N \in(n, \infty)$. A weighted Euclidean space $\left(\mathbb{R}^{n},\|\cdot\|, m\right)$ with $m=e^{-\psi} \operatorname{vol}_{n}, \psi \in C^{\infty}\left(\mathbb{R}^{n}\right)$, satisfies

$$
m((1-t) A+t B)^{1 / N} \geq(1-t) m(A)^{1 / N}+t m(B)^{1 / N}
$$

for all measurable sets $A, B \subset \mathbb{R}^{n}$ and all $t \in[0,1]$ if and only if

$$
\operatorname{Hess} \psi(v, v)-\frac{\langle\nabla \psi(x), v\rangle^{2}}{N-n} \geq 0
$$

holds for all unit vectors $v \in T_{x} \mathbb{R}^{n}$. 
Proof. We first prove that (4.5) implies (4.4). Similarly to Theorem 4.1, we assume that $A$ and $B$ are bounded and of positive measure, and set

$$
\mu_{0}:=\frac{\chi_{A}}{m(A)} m, \quad \mu_{1}:=\frac{\chi_{B}}{m(B)} m .
$$

We again find a convex function $f: \mathbb{R}^{n} \longrightarrow \mathbb{R}$ such that $\mu_{t}:=\left(\mathcal{F}_{t}\right)_{\sharp} \mu_{0}$ is the minimal geodesic from $\mu_{0}$ to $\mu_{1}$, where $\mathcal{F}_{t}:=(1-t) \operatorname{Id}_{\mathbb{R}^{n}}+t \nabla f$. Instead of $\operatorname{det}\left(D \mathcal{F}_{t}\right)$, we consider

$$
\operatorname{det}_{m}\left(D \mathcal{F}_{t}(x)\right):=e^{\psi(x)-\psi\left(\mathcal{F}_{t}(x)\right)} \operatorname{det}\left(D \mathcal{F}_{t}(x)\right) .
$$

The coefficient $e^{\psi(x)-\psi\left(\mathcal{F}_{t}(x)\right)}$ represents the ratio of the weights at $x$ and $\mathcal{F}_{t}(x)$. As in Theorem 4.1 (see, especially, (4.3)) ), it is sufficient to show the concavity of $\operatorname{det}_{m}\left(D \mathcal{F}_{t}(x)\right)^{1 / N}$ to derive the desired inequality (4.4). Fix $x \in A$ and put

$$
\gamma(t):=\mathcal{F}_{t}(x), \quad \Phi_{m}(t):=\operatorname{det}_{m}\left(D \mathcal{F}_{t}(x)\right)^{1 / N}, \quad \Phi(t):=\operatorname{det}\left(D \mathcal{F}_{t}(x)\right)^{1 / n} .
$$

On the one hand, it is proved in (4.3) that $\Phi(t) \geq(1-t) \Phi(0)+t \Phi(1)$. On the other hand, the assumption (4.5) implies that $e^{-\psi\left(\mathcal{F}_{t}(x)\right) /(N-n)}$ is a concave function in $t$. These together imply (4.4) via the Hölder inequality. To be precise, we have

$$
\begin{aligned}
& \Phi_{m}(t)=e^{\left\{\psi(x)-\psi\left(\mathcal{F}_{t}(x)\right)\right\} / N} \Phi(t)^{n / N} \\
& \geq e^{\psi(x) / N}\left\{(1-t) e^{-\psi(x) /(N-n)}+t e^{-\psi\left(\mathcal{F}_{1}(x)\right) /(N-n)}\right\}^{(N-n) / N}\{(1-t) \Phi(0)+t \Phi(1)\}^{n / N},
\end{aligned}
$$

and then the Hölder inequality yields

$$
\begin{aligned}
\Phi_{m}(t) & \geq e^{\psi(x) / N}\left\{(1-t) e^{-\psi(x) / N} \Phi(0)^{n / N}+t e^{-\psi\left(\mathcal{F}_{1}(x)\right) / N} \Phi(1)^{n / N}\right\} \\
& =(1-t) \Phi_{m}(0)+t \Phi_{m}(1) .
\end{aligned}
$$

To see the converse, we fix an arbitrary unit vector $v \in T_{x} \mathbb{R}^{n}$ and set $\gamma(t):=x+t v$ for $t \in \mathbb{R}$ and $a:=\langle\nabla \psi(x), v\rangle /(N-n)$. Given $\varepsilon>0$ and $\delta \in \mathbb{R}$ with $\varepsilon,|\delta| \ll 1$, we consider two open balls (see Figure 6 where $a \delta>0$ )

$$
A_{+}:=B(\gamma(\delta), \varepsilon(1-a \delta)), \quad A_{-}:=B(\gamma(-\delta), \varepsilon(1+a \delta)) .
$$

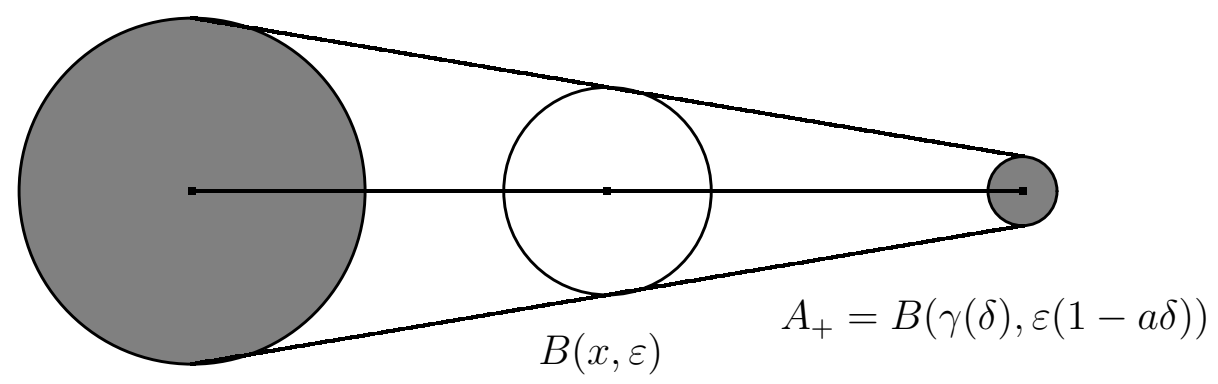

$A_{-}=B(\gamma(-\delta), \varepsilon(1+a \delta))$

Figure 6 
Note that $A_{+}=A_{-}=B(x, \varepsilon)$ for $\delta=0$ and that $(1 / 2) A_{-}+(1 / 2) A_{+}=B(x, \varepsilon)$. We also observe that

$$
m\left(A_{ \pm}\right)=e^{-\psi(\gamma( \pm \delta))} c_{n} \varepsilon^{n}(1 \mp a \delta)^{n}+O\left(\varepsilon^{n+1}\right),
$$

where $c_{n}=\operatorname{vol}_{n}(B(0,1))$ and $O\left(\varepsilon^{n+1}\right)$ is independent of $\delta$. Applying (4.4) to $A_{ \pm}$with $t=1 / 2$, we obtain

$$
m(B(x, \varepsilon)) \geq \frac{1}{2^{N}}\left\{m\left(A_{-}\right)^{1 / N}+m\left(A_{+}\right)^{1 / N}\right\}^{N} .
$$

We know that $m(B(x, \varepsilon))=e^{-\psi(x)} c_{n} \varepsilon^{n}+O\left(\varepsilon^{n+1}\right)$. In order to estimate the right-hand side, we calculate

$$
\begin{aligned}
& \left.\frac{\partial^{2}}{\partial \delta^{2}}\left[e^{-\psi(\gamma(\delta)) / N}(1-a \delta)^{n / N}\right]\right|_{\delta=0} \\
& =\left\{-\frac{\operatorname{Hess} \psi(v, v)}{N}+\frac{\langle\nabla \psi, v\rangle^{2}}{N^{2}}+2 \frac{\langle\nabla \psi, v\rangle}{N} \frac{n}{N} a+\frac{n}{N}\left(\frac{n}{N}-1\right) a^{2}\right\} e^{-\psi(x) / N} \\
& =\left\{-\operatorname{Hess} \psi(v, v)+\frac{\langle\nabla \psi, v\rangle^{2}}{N-n}-\frac{n}{N(N-n)}((N-n) a-\langle\nabla \psi, v\rangle)^{2}\right\} \frac{e^{-\psi(x) / N}}{N} .
\end{aligned}
$$

Due to the choice of $a=\langle\nabla \psi(x), v\rangle /(N-n)$ (as the maximizer), we have

$$
\left.\frac{\partial^{2}}{\partial \delta^{2}}\left[e^{-\psi(\gamma(\delta)) / N}(1-a \delta)^{n / N}\right]\right|_{\delta=0}=\left\{\frac{\langle\nabla \psi(x), v\rangle^{2}}{N-n}-\operatorname{Hess} \psi(v, v)\right\} \frac{e^{-\psi(x) / N}}{N}
$$

Thus we find, by the Taylor expansion of $e^{-\psi(\gamma(\delta)) / N}(1-a \delta)^{n / N}$ at $\delta=0$,

$$
\begin{aligned}
& \frac{m\left(A_{-}\right)^{1 / N}+m\left(A_{+}\right)^{1 / N}}{\left(c_{n} \varepsilon^{n}\right)^{1 / N}} \\
& =2 e^{-\psi(x) / N}-\left\{\operatorname{Hess} \psi(v, v)-\frac{\langle\nabla \psi, v\rangle^{2}}{N-n}\right\} \frac{e^{-\psi(x) / N}}{N} \delta^{2}+O\left(\delta^{4}\right)+O(\varepsilon) .
\end{aligned}
$$

Hence we obtain by letting $\varepsilon$ go to zero in (4.6) that

$$
\begin{aligned}
e^{-\psi(x)} & \geq \frac{1}{2^{N}}\left[2 e^{-\psi(x) / N}-\left\{\operatorname{Hess} \psi(v, v)-\frac{\langle\nabla \psi, v\rangle^{2}}{N-n}\right\} \frac{e^{-\psi(x) / N}}{N} \delta^{2}+O\left(\delta^{4}\right)\right]^{N} \\
& =e^{-\psi(x)}\left[1-\frac{1}{2}\left\{\operatorname{Hess} \psi(v, v)-\frac{\langle\nabla \psi, v\rangle^{2}}{N-n}\right\} \delta^{2}\right]+O\left(\delta^{4}\right) .
\end{aligned}
$$

Therefore we conclude

$$
\operatorname{Hess} \psi(v, v)-\frac{\langle\nabla \psi(x), v\rangle^{2}}{N-n} \geq 0
$$

Applying (4.4) to $A=\{x\}, B=B(x, R)$ and $t=r / R$ implies

$$
\frac{m(B(x, R))}{m(B(x, r))} \leq\left(\frac{R}{r}\right)^{N}
$$

for all $x \in \mathbb{R}^{n}$ and $0<r<R$. Thus, compared with Theorem 3.2, $\left(\mathbb{R}^{n},\|\cdot\|, m\right)$ satisfying (4.5) behaves like an ' $N$-dimensional' space of nonnegative Ricci curvature (see Theorem 6.3 for more general theorem in terms of the curvature-dimension condition). 


\subsection{Characterizing lower Ricci curvature bounds}

Now we switch to the weighted Riemannian situation $(M, g, m)$, where $m=e^{-\psi} \operatorname{vol}_{g}$ with $\psi \in C^{\infty}(M)$. Ricci curvature controls $\operatorname{vol}_{n}$ as we saw in Section 3, and Theorem 4.3 suggests that the quantity

$$
\operatorname{Hess} \psi(v, v)-\frac{\langle\nabla \psi, v\rangle^{2}}{N-n}
$$

has an essential information in controlling the effect of the weight. Their combination indeed gives the weighted Ricci curvature as follows (cf. [BE], Qi], [Lo1]).

Definition 4.4 (Weighted Ricci curvature) Given a unit tangent vector $v \in T_{x} M$ and $N \in[n, \infty]$, the weighted Ricci curvature $\operatorname{Ric}_{N}(v)$ is defined by

(1) $\operatorname{Ric}_{n}(v):= \begin{cases}\operatorname{Ric}(v)+\operatorname{Hess} \psi(v, v) & \text { if }\langle\nabla \psi(x), v\rangle=0 \\ -\infty & \text { otherwise }\end{cases}$

(2) $\operatorname{Ric}_{N}(v):=\operatorname{Ric}(v)+\operatorname{Hess} \psi(v, v)-\frac{\langle\nabla \psi(x), v\rangle^{2}}{N-n}$ for $N \in(n, \infty)$;

(3) $\operatorname{Ric}_{\infty}(v):=\operatorname{Ric}(v)+\operatorname{Hess} \psi(v, v)$.

We say that $\operatorname{Ric}_{N} \geq K$ holds for $K \in \mathbb{R}$ if $\operatorname{Ric}_{N}(v) \geq K$ holds for all unit vectors $v \in T M$.

Note that $\operatorname{Ric}_{N} \leq \operatorname{Ric}_{N^{\prime}}$ holds for $n \leq N \leq N^{\prime}<\infty$. Ric $\infty$ is also called the BakryÉmery tensor. If the weight is trivial in the sense that $\psi$ is constant, then $\operatorname{Ric}_{N}$ coincides with Ric for all $N \in[n, \infty]$. One of the most important examples possessing nontrivial weight is the following.

Example 4.5 (Euclidean spaces with log-concave measures) Consider a weighted Euclidean space $\left(\mathbb{R}^{n},\|\cdot\|, m\right)$ with $m=e^{-\psi} \operatorname{vol}_{n}, \psi \in C^{\infty}\left(\mathbb{R}^{n}\right)$. Then clearly $\operatorname{Ric}_{\infty}(v)=$ Hess $\psi(v, v)$, thus $\operatorname{Ric}_{\infty} \geq 0$ if $\psi$ is convex. The most typical and important example satisfying $\operatorname{Ric}_{\infty} \geq K>0$ is the Gausssian measure

$$
m=\left(\frac{K}{2 \pi}\right)^{n / 2} e^{-K\|x\|^{2} / 2} \operatorname{vol}_{n}, \quad \psi(x)=\frac{K}{2}\|x\|^{2}+\frac{n}{2} \log \left(\frac{2 \pi}{K}\right) .
$$

Note that Hess $\psi \geq K$ holds independently of the dimension $n$.

Before stating the main theorem of the section, we mention that optimal transport in a Riemannian manifold is described in the same manner as the Euclidean spaces (due to [Mc2], [CMS1]). Given $\mu_{0}, \mu_{1} \in \mathcal{P}_{c}(M)$ with $\mu_{0}=\rho_{0} \operatorname{vol}_{n} \in \mathcal{P}^{\mathrm{ac}}\left(M, \operatorname{vol}_{n}\right)$, there is a $\left(d^{2} / 2\right)$-convex function $f: M \longrightarrow \mathbb{R}$ such that $\mu_{t}:=\left(\mathcal{F}_{t}\right)_{\sharp} \mu_{0}$ with $\mathcal{F}_{t}(x):=\exp _{x}[t \nabla f(x)]$, $t \in[0,1]$, gives the unique minimal geodesic from $\mu_{0}$ to $\mu_{1}$. We do not give the definition of $\left(d^{2} / 2\right)$-convex functions, but only remark that they are twice differentiable a.e. Furthermore, the absolute continuity of $\mu_{0}$ implies that $\mu_{t}$ is absolutely continuous for all $t \in[0,1)$, so that we can set $\mu_{t}=\rho_{t} m$. Since $\mathcal{F}_{t}$ is differentiable $\mu_{0}$-a.e., we can consider the Jacobian $\left\|\left(D \mathcal{F}_{t}\right)_{x}\right\|$ (with respect to $\operatorname{vol}_{n}$ ) which satisfies the Monge-Ampère equation

$$
\rho_{0}(x)=\rho_{t}\left(\mathcal{F}_{t}(x)\right)\left\|\left(D \mathcal{F}_{t}\right)_{x}\right\|
$$


for $\mu_{0}$-a.e. $x$.

We next introduce two entropy functionals. Given $N \in[n, \infty)$ and an absolutely continuous probability measure $\mu=\rho m \in \mathcal{P}^{\text {ac }}(M, m)$, we define the Rényi entropy as

$$
S_{N}(\mu):=-\int_{M} \rho^{1-1 / N} d m
$$

We also define the relative entropy with respect to the reference measure $m$ by

$$
\operatorname{Ent}_{m}(\mu):=\int_{M} \rho \log \rho d m
$$

Note that Ent $_{m}$ has the opposite sign to the Boltzmann entropy. The domain of these functionals will be extended in the next section ((5.1), (5.2) ) to probability measures possibly with nontrivial singular part. In this section, however, we consider only absolutely continuous measures for the sake of simplicity. As any two points in $\mathcal{P}_{c}^{\text {ac }}(M, m)$ are connected by a unique minimal geodesic contained in $\mathcal{P}_{c}^{\text {ac }}(M, m)$, the convexity of $S_{N}$ and Ent $_{m}$ in $\mathcal{P}_{c}^{\text {ac }}(M, m)$ makes sense.

Recall (2.3) for the definition of the function $\beta_{K, N}^{t}$. The following theorem is due to von Renesse, Sturm and many others, see Further Reading for more details.

Theorem 4.6 (A characterization of Ricci curvature bound) For a weighted Riemannian manifold $(M, g, m)$ with $m=e^{-\psi} \operatorname{vol}_{g}, \psi \in C^{\infty}(M)$, we have $\operatorname{Ric}_{N} \geq K$ for some $K \in \mathbb{R}$ and $N \in[n, \infty)$ if and only if any pair of measures $\mu_{0}=\rho_{0} m, \mu_{1}=\rho_{1} m \in$ $\mathcal{P}_{c}^{\mathrm{ac}}(M, m)$ satisfies

$$
\begin{aligned}
S_{N}\left(\mu_{t}\right) \leq & -(1-t) \int_{M \times M} \beta_{K, N}^{1-t}(d(x, y))^{1 / N} \rho_{0}(x)^{-1 / N} d \pi(x, y) \\
& -t \int_{M \times M} \beta_{K, N}^{t}(d(x, y))^{1 / N} \rho_{1}(y)^{-1 / N} d \pi(x, y)
\end{aligned}
$$

for all $t \in(0,1)$, where $\left(\mu_{t}\right)_{t \in[0,1]} \subset \mathcal{P}_{c}^{\text {ac }}(M, m)$ is the unique minimal geodesic from $\mu_{0}$ to $\mu_{1}$ in the $L^{2}$-Wasserstein space $\left(\mathcal{P}_{2}(M), d_{2}^{W}\right)$, and $\pi$ is the unique optimal coupling of $\mu_{0}$ and $\mu_{1}$.

Similarly, $\operatorname{Ric}_{\infty} \geq K$ is equivalent to

$$
\operatorname{Ent}_{m}\left(\mu_{t}\right) \leq(1-t) \operatorname{Ent}_{m}\left(\mu_{0}\right)+t \operatorname{Ent}_{m}\left(\mu_{1}\right)-\frac{K}{2}(1-t) t d_{2}^{W}\left(\mu_{0}, \mu_{1}\right)^{2} .
$$

Outline of proof. We give a sketch of the proof for $N<\infty$ along the lines of [St4] and [LV1]. The case of $N=\infty$ goes along the essentially same line.

First, we assume $\operatorname{Ric}_{N} \geq K$. Fix $\mu_{0}=\rho_{0} m, \mu_{1}=\rho_{1} m \in \mathcal{P}_{c}^{\mathrm{ac}}(M, m)$ and take a $\left(d^{2} / 2\right)$-convex function $f: M \longrightarrow \mathbb{R}$ such that $\mathcal{F}_{t}(x):=\exp _{x}[t \nabla f(x)], t \in[0,1]$, provides the unique minimal geodesic $\mu_{t}=\rho_{t} m=\left(\mathcal{F}_{t}\right)_{\sharp} \mu_{0}$ from $\mu_{0}$ to $\mu_{1}$. Taking the weight $e^{-\psi}$ into account, we introduce the Jacobian $\mathbf{J}_{t}^{\psi}(x):=e^{\psi(x)-\psi\left(\mathcal{F}_{t}(x)\right)}\left\|\left(D \mathcal{F}_{t}\right)_{x}\right\|$ with respect to $m$ (like $\operatorname{det}_{m}$ in Theorem 4.3). Then it follows from the Monge-Ampère equation (4.8) with respect to $\mathrm{vol}_{n}$ that

$$
\rho_{0}(x)=\rho_{t}\left(\mathcal{F}_{t}(x)\right) \mathbf{J}_{t}^{\psi}(x)
$$


for $\mu_{0}$-a.e. $x$ (i.e., the Monge-Ampère equation with respect to $m$ ).

Now, the essential point is that optimal transport is performed along geodesics $t \longmapsto$ $\exp _{x}[t \nabla f(x)]=\mathcal{F}_{t}(x)$. Therefore its variational vector fields are Jacobi fields (recall (3.1)), and controlled by Ricci curvature. Together with the weight control as in Theorem 4.3, calculations somewhat similar to (but more involved than) Theorem 3.2 shows our key inequality

$$
\mathbf{J}_{t}^{\psi}(x)^{1 / N} \geq(1-t) \beta_{K, N}^{1-t}\left(d\left(x, \mathcal{F}_{1}(x)\right)\right)^{1 / N}+t \beta_{K, N}^{t}\left(d\left(x, \mathcal{F}_{1}(x)\right)\right)^{1 / N} \mathbf{J}_{1}^{\psi}(x)^{1 / N} .
$$

This inequality can be thought of as an infinitesimal version of the Brunn-Minkowski inequality (see (4.4) and Theorem 6.1(i) as well). As the change of variables formula and the Monge-Ampère equation (4.13) yield

$$
S_{N}\left(\mu_{t}\right)=-\int_{M} \rho_{t}\left(\mathcal{F}_{t}\right)^{1-1 / N} \mathbf{J}_{t}^{\psi} d m=-\int_{M}\left(\frac{\mathbf{J}_{t}^{\psi}}{\rho_{0}}\right)^{1 / N} d \mu_{0},
$$

we obtain from (4.14) (and (4.13) again) that

$$
\begin{aligned}
S_{N}\left(\mu_{t}\right) \leq & -(1-t) \int_{M} \frac{\beta_{K, N}^{1-t}\left(d\left(x, \mathcal{F}_{1}(x)\right)\right)^{1 / N}}{\rho_{0}(x)^{1 / N}} d \mu_{0}(x) \\
& -t \int_{M} \frac{\beta_{K, N}^{t}\left(d\left(x, \mathcal{F}_{1}(x)\right)\right)^{1 / N}}{\rho_{1}\left(\mathcal{F}_{1}(x)\right)^{1 / N}} d \mu_{0}(x) .
\end{aligned}
$$

This is the desired inequality (4.11), for $\pi=\left(\operatorname{Id}_{M} \times \mathcal{F}_{1}\right)_{\sharp} \mu_{0}$.

Second, we assume (4.11). Then applying it to uniform distributions on balls (as in the proof of Theorem 4.3) shows $\operatorname{Ric}_{N} \geq K$. More precisely, we use the generalized Brunn-Minkowski inequality (Theorem 6.1) instead of (4.4).

As $\beta_{0, N}^{t} \equiv 1$, the inequality (4.11) is simplified into the convexity of $S_{N}$

$$
S_{N}\left(\mu_{t}\right) \leq(1-t) S_{N}\left(\mu_{0}\right)+t S_{N}\left(\mu_{1}\right)
$$

when $K=0$. For $K \neq 0$, however, the $K$-convexity of $S_{N}$

$$
S_{N}\left(\mu_{t}\right) \leq(1-t) S_{N}\left(\mu_{0}\right)+t S_{N}\left(\mu_{1}\right)-\frac{K}{2}(1-t) t d_{2}^{W}\left(\mu_{0}, \mu_{1}\right)^{2}
$$

turns out uninteresting (see [St2, Theorem 1.3]). This is a reason why we need to consider a more subtle inequality like (4.11).

Theorem 4.6 gives an answer to Question 3.5, for the conditions (4.11), (4.12) are written in terms of only distance and measure, without using the differentiable structure. Then it is interesting to consider these conditions for general metric measure spaces as 'synthetic Ricci curvature bounds', and we should verify the stability. We discuss them in the next section.

Further Reading We refer to [AGS], Vi1] and [Vi2, Part I] for the basics of optimal transport theory and Wasserstein geometry. McCann's [Mc2] fundamental result on the 
shape of optimal transport maps is generalized to not necessarily compactly supported measures in [FF] and [FG] (see also [Vi2, Chapter 10]).

See [Ga] and [Le, Section 2.2] for the Brunn-Minkowski inequality and related topics. The Bakry-Émery tensor Ric $\infty$ was introduced in [BE], and its generalization $\operatorname{Ric}_{N}$ is due to Qian Qi]. See also [Lo1] for geometric and topological applications, [Mo, Chapter 18] and the references therein for minimal surface theory in weighted manifolds (which are called manifolds with density there).

After McCann's [Mc1] pinoneering work on the convexity of the relative entropy along geodesics in the Wasserstein space (called the displacement convexity) over Euclidean spaces, Cordero-Erausquin, McCann and Schmuckenschläger [CMS1] first showed that Ric $\geq 0$ implies (4.12) with $K=0$ in unweighted Riemannian manifolds. They [CMS2] further proved that Ric $_{\infty} \geq K$ implies (4.12) in the weighted situation. Then Theorem 4.6 is due to von Renesse and Sturm vRS], [St2] for $N=\infty$, and independently to Sturm [St3], [St4] and Lott and Villani [LV2], LV1] for $N<\infty$.

We comment on recent work on a variant of (4.11). Studied in [BaS1] is the following inequality (called the reduced curvature-dimension condition)

$$
\begin{aligned}
S_{N}\left(\mu_{t}\right) \leq & -(1-t) \int_{M \times M} \beta_{K, N+1}^{1-t}(d(x, y))^{1 / N} \rho_{0}(x)^{-1 / N} d \pi(x, y) \\
& -t \int_{M \times M} \beta_{K, N+1}^{t}(d(x, y))^{1 / N} \rho_{1}(y)^{-1 / N} d \pi(x, y) .
\end{aligned}
$$

Note the difference between

$$
t \beta_{K, N}^{t}(r)^{1 / N}=t^{1 / N}\left(\frac{\mathbf{s}_{K, N}(t r)}{\mathbf{s}_{K, N}(r)}\right)^{1-1 / N}, \quad t \beta_{K, N+1}^{t}(r)^{1 / N}=\frac{\mathbf{s}_{K, N+1}(t r)}{\mathbf{s}_{K, N+1}(r)} .
$$

We remark that (4.15) coincides with (4.11) when $K=0$, and is weaker than (4.11) for general $K \neq 0$. The condition (4.15) is also equivalent to $\operatorname{Ric}_{N} \geq K$ for Riemannian manifolds. In the setting of metric measure spaces, (4.15) has some advantages such as the tensorization and the localization properties (see Subsection 8.3 for more details). One drawback is that, as it is weaker than (4.11), (4.15) derives slightly worse estimates than (4.11) (in the Bishop-Gromov volume comparison (Theorem 6.3), the Bonnet-Myers diameter bound (Theorem 6.5), the Lichnerowicz inequality (Theorem 6.7) etc.). Nevertheless, such weaker estimates are sufficient for several topological applications.

See also [St2] and [OT] for the $K$-convexity of generalized entropies (or free energies) and its characterization and applications. It is discussed in [St2, Theorem 1.7] that there is a class of functionals whose $K$-convexity is equivalent to $\operatorname{Ric} \geq K$ and $\operatorname{dim} \leq N$ for unweighted Riemannian manifolds. The choice of a functional is by no means unique, and it is unclear how this observation relates to the curvature-dimension condition.

\section{The curvature-dimension condition and stability}

Motivated by Theorem 4.6, we introduce the curvature-dimension condition for metric measure spaces and show that it is stable under the measured Gromov-Hausdorff convergence. In this and the next sections, $(X, d, m)$ will always be a metric measure space in the sense of Section 2 , 


\subsection{The curvature-dimension condition}

We can regard the conditions (4.11), (4.12) as convexity estimates of the functionals $S_{N}$ and $\mathrm{Ent}_{m}$. For the sake of consistency with the monotonicity of $\operatorname{Ric}_{N}$ in $N\left(\operatorname{Ric}_{N} \leq \mathrm{Ric}_{N^{\prime}}\right.$ for $N \leq N^{\prime}$ ), we introduce important classes of functionals (due to McCann [Mc1]) including $S_{N}$ and Ent $_{m}$.

For $N \in[1, \infty)$, denote by $\mathcal{D C}_{N}$ (displacement convexity class) the set of continuous convex functions $U:[0, \infty) \longrightarrow \mathbb{R}$ such that $U(0)=0$ and that the function $\varphi(s)=$ $s^{N} U\left(s^{-N}\right)$ is convex on $(0, \infty)$. Similarly, define $\mathcal{D} \mathcal{C}_{\infty}$ as the set of continuous convex functions $U:[0, \infty) \longrightarrow \mathbb{R}$ such that $U(0)=0$ and that $\varphi(s)=e^{s} U\left(e^{-s}\right)$ is convex on $\mathbb{R}$. In both cases, the convexity of $U$ implies that $\varphi$ is non-increasing. Observe the monotonicity, $\mathcal{D C}_{N^{\prime}} \subset \mathcal{D} \mathcal{C}_{N}$ holds for $1 \leq N \leq N^{\prime} \leq \infty$.

For $\mu \in \mathcal{P}(X)$, using its Lebesgue decomposition $\mu=\rho m+\mu^{s}$ into absolutely continuous and singular parts, we set

$$
U_{m}(\mu):=\int_{X} U(\rho) d m+U^{\prime}(\infty) \mu^{s}(X), \quad U^{\prime}(\infty):=\lim _{r \rightarrow \infty} \frac{U(r)}{r} .
$$

Note that $U^{\prime}(\infty)$ indeed exists as $U(r) / r$ is non-decreasing. In the case where $U^{\prime}(\infty)=\infty$, we set $\infty \cdot 0:=0$ by convention. The most important element of $\mathcal{D C}_{N}$ is the function $U(r)=N r\left(1-r^{-1 / N}\right)$ which induces the Rényi entropy (4.9) in a slightly deformed form as

$$
U_{m}(\mu)=N \int_{X} \rho\left(1-\rho^{-1 / N}\right) d m+N \mu^{s}(X)=N\left(1-\int_{X} \rho^{1-1 / N} d m\right) .
$$

This extends (4.9) to whole $\mathcal{P}(X)$. Letting $N$ go to infinity, we have $U(r)=r \log r \in \mathcal{D} \mathcal{C}_{\infty}$ as well as the relative entropy (extending (4.10) )

$$
U_{m}(\mu)=\int_{X} \rho \log \rho d m+\infty \cdot \mu^{s}(X) .
$$

Let us denote by $\Gamma(X)$ the set of minimal geodesics $\gamma:[0,1] \longrightarrow X$ endowed with the distance

$$
d_{\Gamma(X)}\left(\gamma_{1}, \gamma_{2}\right):=\sup _{t \in[0,1]} d_{X}\left(\gamma_{1}(t), \gamma_{2}(t)\right) .
$$

Define the evaluation map $e_{t}: \Gamma(X) \longrightarrow X$ for $t \in[0,1]$ as $e_{t}(\gamma):=\gamma(t)$, and note that this is 1-Lipschitz. A probability measure $\Pi \in \mathcal{P}(\Gamma(X))$ is called a dynamical optimal transference plan if the curve $\alpha(t):=\left(e_{t}\right)_{\sharp} \Pi, t \in[0,1]$, is a minimal geodesic in $\left(\mathcal{P}_{2}(X), d_{2}^{W}\right)$. Then $\pi:=\left(e_{0} \times e_{1}\right)_{\sharp} \Pi$ is an optimal coupling of $\alpha(0)$ and $\alpha(1)$. We remark that $\Pi$ is not uniquely determined by $\alpha$ and $\pi$, that is to say, different plans $\Pi$ and $\Pi^{\prime}$ could generate the same minimal geodesic $\alpha$ and optimal coupling $\pi$. If $(X, d)$ is locally compact (and hence proper), then any minimal geodesic in $\mathcal{P}_{2}(X)$ is associated with a (not necessarily unique) dynamical optimal transference plan ([LV2, Proposition 2.10], [Vi2, Corollary 7.22]).

Now we are ready to present the precise definition of the curvature-dimension condition in the form due to Lott and Villani (after Sturm and others, see Further Reading of this and the previous sections). 
Definition 5.1 (The curvature-dimension condition) Suppose that $m(B(x, r)) \in$ $(0, \infty)$ holds for all $x \in X$ and $r \in(0, \infty)$. For $K \in \mathbb{R}$ and $N \in(1, \infty]$, we say that a metric measure space $(X, d, m)$ satisfies the curvature-dimension condition $\operatorname{CD}(K, N)$ if, for any $\mu_{0}=\rho_{0} m+\mu_{0}^{s}, \mu_{1}=\rho_{1} m+\mu_{1}^{s} \in \mathcal{P}_{b}(X)$, there exists a dynamical optimal transference plan $\Pi \in \mathcal{P}(\Gamma(X))$ associated with a minimal geodesic $\alpha(t)=\left(e_{t}\right)_{\sharp} \Pi, t \in[0,1]$, from $\mu_{0}$ to $\mu_{1}$ and an optimal coupling $\pi=\left(e_{0} \times e_{1}\right)_{\sharp} \Pi$ of $\mu_{0}$ and $\mu_{1}$ such that we have

$$
\begin{aligned}
U_{m}(\alpha(t)) \leq & (1-t) \int_{X \times X} \beta_{K, N}^{1-t}(d(x, y)) U\left(\frac{\rho_{0}(x)}{\beta_{K, N}^{1-t}(d(x, y))}\right) d \pi_{x}(y) d m(x) \\
& +t \int_{X \times X} \beta_{K, N}^{t}(d(x, y)) U\left(\frac{\rho_{1}(y)}{\beta_{K, N}^{t}(d(x, y))}\right) d \pi_{y}(x) d m(y) \\
& +U^{\prime}(\infty)\left\{(1-t) \mu_{0}^{s}(X)+t \mu_{1}^{s}(X)\right\}
\end{aligned}
$$

for all $U \in \mathcal{D} \mathcal{C}_{N}$ and $t \in(0,1)$, where $\pi_{x}$ and $\pi_{y}$ denote disintegrations of $\pi$ by $\mu_{0}$ and $\mu_{1}$, i.e., $d \pi(x, y)=d \pi_{x}(y) d \mu_{0}(x)=d \pi_{y}(x) d \mu_{1}(y)$.

In the special case of $K=0$, as $\beta_{0, N}^{t} \equiv 1$, the inequality (5.3) means the convexity of $U_{m}$

$$
U_{m}(\alpha(t)) \leq(1-t) U_{m}\left(\mu_{0}\right)+t U_{m}\left(\mu_{1}\right),
$$

without referring to the optimal coupling $\pi$. In the case where both $\mu_{0}$ and $\mu_{1}$ are absolutely continuous, we have $d \pi(x, y)=\rho_{0}(x) d \pi_{x}(y) d m(x)=\rho_{1}(y) d \pi_{y}(x) d m(y)$ and hence (5.3) is rewritten in a more symmetric form as

$$
\begin{aligned}
U_{m}(\alpha(t)) \leq & (1-t) \int_{X \times X} \frac{\beta_{K, N}^{1-t}(d(x, y))}{\rho_{0}(x)} U\left(\frac{\rho_{0}(x)}{\beta_{K, N}^{1-t}(d(x, y))}\right) d \pi(x, y) \\
& +t \int_{X \times X} \frac{\beta_{K, N}^{t}(d(x, y))}{\rho_{1}(y)} U\left(\frac{\rho_{1}(y)}{\beta_{K, N}^{t}(d(x, y))}\right) d \pi(x, y) .
\end{aligned}
$$

Note that choosing $U(r)=N r\left(1-r^{-1 / N}\right)$ and $U(r)=r \log r$ in (5.4) reduce to (4.11) and (4.12), respectively. We summarize remark on and the background of Definition 5.1.

Remark 5.2 (a) It is easily checked that, if $(X, d, m)$ satisfies $\operatorname{CD}(K, N)$, then the scaled metric measure space $\left(X, c d, c^{\prime} m\right)$ for $c, c^{\prime}>0$ satisfies $\operatorname{CD}\left(K / c^{2}, N\right)$.

(b) In Definition 5.1, to be precise, we need to impose the condition

$$
m(X \backslash B(x, \pi \sqrt{(N-1) / K}))=0
$$

for all $x \in X$ if $K>0$ and $N<\infty$, in order to stay inside the domain of $\beta_{K, N}^{t}$. Nevertheless, this is always the case by virtue of the generalized Bonnet-Myers theorem (Theorem 6.5) below.

(c) Recall that $U(r) / r$ is non-decreasing, and observe that $\beta_{K, N}^{t}(r)$ is increasing in $K$ and decreasing in $N$. Combining this with the monotonicity $\mathcal{D} \mathcal{C}_{N^{\prime}} \subset \mathcal{D C} \mathcal{C}_{N}$ for $N \leq N^{\prime}$ (and (b) above), we see that $\operatorname{CD}(K, N)$ implies $\operatorname{CD}\left(K^{\prime}, N^{\prime}\right)$ for all $K^{\prime} \leq K$ and $N^{\prime} \geq N$. Therefore, in the condition $\operatorname{CD}(K, N), K$ represents a lower bound of the Ricci curvature and $N$ represents an upper bound of the dimension. 
(d) The validity of (5.3) along only 'some' geodesic is essential to establish the stability. In fact, if we impose it for all geodesics between $\mu_{0}$ and $\mu_{1}$, then it is not stable under convergence (in the sense of Theorem 5.6). This is because, when a sequence $\left\{\left(X_{i}, d_{i}\right)\right\}_{i \in \mathbb{N}}$ converges to the limit space $(X, d)$, there may be a geodesic in $X$ which can not be represented as the limit of a sequence of geodesics in $X_{i}$. Therefore the convexity along geodesics in $X_{i}$ does not necessarily imply the convexity along all geodesics in $X$. One typical example is a sequence of $\ell_{p}^{n}$-spaces as $p$ goes to 1 or $\infty$. Only straight lines are geodesics in $\ell_{p}^{n}$ with $1<p<\infty$, while $\ell_{1}^{n}$ and $\ell_{\infty}^{n}$ have much more geodesics. (See Figure 7, where $\gamma_{i}$ for all $i=0, \ldots, 3$ are geodesic for $\ell_{\infty}^{2}$, while only the straight line segment $\gamma_{0}$ is geodesic for $\ell_{p}^{2}$ with $1<p<\infty$.) In fact, $\ell_{p}^{n}$ equipped with the Lebesgue measure satisfies $\mathrm{CD}(0, n)$ for all $1<p<\infty$ (Example 7.4(a)), but $\ell_{1}^{n}$ and $\ell_{\infty}^{n}$ do not satisfy $\operatorname{CD}(0, n)$.

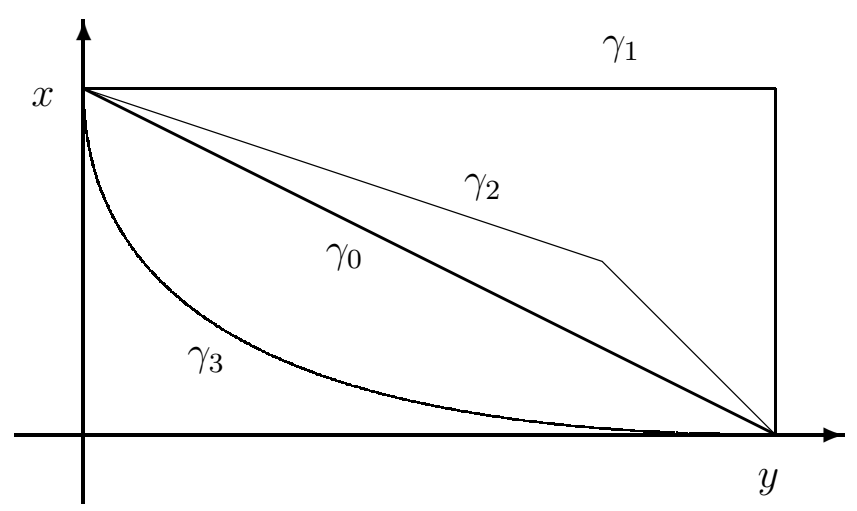

Figure 7

(e) In Riemannian manifolds or, more generally, non-branching proper metric measure spaces, we can reduce (5.3) to a special case from two aspects as follows. If (5.4) holds for $U(r)=N r\left(1-r^{-1 / N}\right)$ (or $U(r)=r \log r$ if $N=\infty$ ) and all measures in $\mathcal{P}_{b}^{\text {ac }}(X, m)$ (and hence in $\mathcal{P}_{c}^{\mathrm{ac}}(X, m)$ ) with continuous densities, then (5.3) holds for all $U \in \mathcal{D} \mathcal{C}_{N}$ and all measures in $\mathcal{P}_{b}(X)$ ([St4, Proposition 4.2], [LV2, Proposition 3.21, Lemma 3.24]). In this sense, (4.11) and (4.12) are essential among the class of inequalities (5.3). A geodesic space is said to be non-branching if geodesics in it do not branch (see Subsection 8.1 for the precise definition).

(f) In Riemannian manifolds, (4.14) implies (5.4) for all $U \in \mathcal{D} \mathcal{C}_{N}$. Indeed, $\alpha(t)=\rho_{t} m$ is absolutely continuous and the change of variables formula and the Monge-Ampère equation (4.13) imply

$$
U_{m}(\alpha(t))=\int_{M} U\left(\rho_{t}\right) d m=\int_{M} U\left(\rho_{t}\left(\mathcal{F}_{t}\right)\right) \mathbf{J}_{t}^{\psi} d m=\int_{M} U\left(\frac{\rho_{0}}{\mathbf{J}_{t}^{\psi}}\right) \frac{\mathbf{J}_{t}^{\psi}}{\rho_{0}} d \mu_{0} .
$$

For $N<\infty$, as $\varphi(s)=s^{N} U\left(s^{-N}\right)$ is non-increasing and convex, (4.14) yields

$$
\begin{aligned}
& U_{m}(\alpha(t)) \leq \int_{M} \varphi\left((1-t) \frac{\beta_{K, N}^{1-t}\left(d\left(x, \mathcal{F}_{1}(x)\right)\right)^{1 / N}}{\rho_{0}(x)^{1 / N}}+t \frac{\beta_{K, N}^{t}\left(d\left(x, \mathcal{F}_{1}(x)\right)\right)^{1 / N}}{\rho_{1}\left(\mathcal{F}_{1}(x)\right)^{1 / N}}\right) d \mu_{0}(x) \\
& \leq \int_{M}\left\{(1-t) \varphi\left(\frac{\beta_{K, N}^{1-t}\left(d\left(x, \mathcal{F}_{1}(x)\right)\right)^{1 / N}}{\rho_{0}(x)^{1 / N}}\right)+t \varphi\left(\frac{\beta_{K, N}^{t}\left(d\left(x, \mathcal{F}_{1}(x)\right)\right)^{1 / N}}{\rho_{1}\left(\mathcal{F}_{1}(x)\right)^{1 / N}}\right)\right\} d \mu_{0}(x) .
\end{aligned}
$$


The case of $N=\infty$ is similar. This means that the infinitesimal expression of $\operatorname{CD}(K, N)$ is always (4.14) whatever $U$ is, and various ways of integration give rise to the definition of $\mathrm{CD}(K, N)$ involving $\mathcal{D} \mathcal{C}_{N}$.

Although $\mathbf{J}_{t}^{\psi}$ relies on the differentiable structure of $M$, it is possible to rewrite (4.14) through (4.13) as

$$
\begin{aligned}
\rho_{t}\left(\mathcal{F}_{t}(x)\right)^{-1 / N} \geq & (1-t) \beta_{K, N}^{1-t}\left(d\left(x, \mathcal{F}_{1}(x)\right)\right)^{1 / N} \rho_{0}(x)^{-1 / N} \\
& +t \beta_{K, N}^{t}\left(d\left(x, \mathcal{F}_{1}(x)\right)\right)^{1 / N} \rho_{1}\left(\mathcal{F}_{1}(x)\right)^{-1 / N}
\end{aligned}
$$

(see [St4, Proposition 4.2(iv)]). This makes sense in metric measure spaces, however, the integrated inequalities (i.e., (5.3), (5.4) ) are more convenient for verifying the stability.

(g) The role of the dynamical optimal transference plan $\Pi$ may seem unclear in Definition 5.1, as only $\alpha$ and $\pi$ appear in (5.3). We use only $\alpha$ and $\pi$ also in applications in Section 6. As we shall see in Theorem 5.6, it is the stability in which $\Pi$ plays a crucial role.

\subsection{Stability and geometric background}

As we mentioned in Remark 3.1, one geometric motivation behind the curvature-dimension condition is the theory of Alexandrov spaces. That is to say, we would like to find a way of formulating and investigating singular spaces of Ricci curvature bounded below in some sense (recall Question 3.5). Then, what kind of singular spaces should we consider? Here comes into play another deep theory of the precompactness with respect to the convergence of metric (measure) spaces. Briefly speaking, the precompactness ensures that a sequence of Riemannian manifolds with a uniform lower Ricci curvature bound contains a convergent subsequence. Such a limit space is not a manifold any more, but should inherits some properties. In order to make use of the curvature-dimension condition in the limit, we need to establish that it is preserved under the convergence (actually, the limit of Alexandrov spaces is again an Alexandrov space.)

We say that a map $\varphi: Y \longrightarrow X$ between metric spaces is $\varepsilon$-approximating for $\varepsilon>0$ if

$$
\left|d_{X}(\varphi(y), \varphi(z))-d_{Y}(y, z)\right| \leq \varepsilon
$$

holds for all $y, z \in Y$ and if $\overline{B(\varphi(Y), \varepsilon)}=X$.

Definition 5.3 (Measured Gromov-Hausdorff convergence) Consider a sequence of metric measure spaces $\left\{\left(X_{i}, d_{i}, m_{i}\right)\right\}_{i \in \mathbb{N}}$ and another metric measure space $(X, d, m)$.

(1) (Compact case) Assume that $\left(X_{i}, d_{i}\right)$ for all $i \in \mathbb{N}$ and $(X, d)$ are compact. We say that $\left\{\left(X_{i}, d_{i}, m_{i}\right)\right\}_{i \in \mathbb{N}}$ converges to $(X, d, m)$ in the sense of the measured GromovHausdorff convergence if there are sequences of positive numbers $\left\{\varepsilon_{i}\right\}_{i \in \mathbb{N}}$ and Borel $\operatorname{maps}\left\{\varphi_{i}: X_{i} \longrightarrow X\right\}_{i \in \mathbb{N}}$ such that $\lim _{i \rightarrow \infty} \varepsilon_{i}=0, \varphi_{i}$ is an $\varepsilon_{i}$-approximating map, and that $\left(\varphi_{i}\right)_{\sharp} m_{i}$ weakly converges to $m$.

(2) (Noncompact case) Assume that $\left(X_{i}, d_{i}\right)$ for all $i \in \mathbb{N}$ and $(X, d)$ are proper, and fix base points $x_{i} \in X_{i}$ and $x \in X$. We say that $\left\{\left(X_{i}, d_{i}, m_{i}, x_{i}\right)\right\}_{i \in \mathbb{N}}$ converges to 
$(X, d, m, x)$ in the sense of the pointed measured Gromov-Hausdorff convergence if, for all $R>0,\left\{\left(\overline{B\left(x_{i}, R\right)}, d_{i}, m_{i}\right)\right\}_{i \in \mathbb{N}}$ converges to $(\overline{B(x, R)}, d, m)$ in the sense of the measured Gromov-Hausdorff convergence (as in (1) above).

If we consider only distance structures $\left(X_{i}, d_{i}\right)$ and $(X, d)$ and remove the weak convergence condition on $\varphi_{i}$, then it is the (pointed) Gromov-Hausdorff convergence under which the lower sectional curvature bound in the sense of Alexandrov is known to be preserved. The following observation ([LV2, Proposition 4.1]) says that the Gromov-Hausdorff convergence of a sequence of metric spaces is propagated to the Wasserstein spaces over them.

Proposition 5.4 If a sequence of compact metric spaces $\left\{\left(X_{i}, d_{i}\right)\right\}_{i \in \mathbb{N}}$ converges to a compact metric space $(X, d)$ in the sense of the Gromov-Hausdorff convergence, then so does the sequence of Wasserstein spaces $\left\{\left(\mathcal{P}\left(X_{i}\right), d_{2}^{W}\right)\right\}_{i \in \mathbb{N}}$ to $\left(\mathcal{P}(X), d_{2}^{W}\right)$.

More precisely, $\varepsilon_{i}$-approximating maps $\varphi_{i}: X_{i} \longrightarrow X$ give rise to $\theta\left(\varepsilon_{i}\right)$-approximating maps $\left(\varphi_{i}\right)_{\sharp}: \mathcal{P}\left(X_{i}\right) \longrightarrow \mathcal{P}(X)$ such that $\theta$ is a universal function satisfying $\lim _{\varepsilon \downarrow 0} \theta(\varepsilon)=0$.

In the noncompact case, the pointed Gromov-Hausdorff convergence of $\left\{\left(X_{i}, d_{i}, x_{i}\right)\right\}_{i \in \mathbb{N}}$ to $(X, d, x)$ similarly implies the Gromov-Hausdorff convergence of $\left\{\left(\mathcal{P}\left(\overline{B\left(x_{i}, R\right)}\right), d_{2}^{W}\right)\right\}_{i \in \mathbb{N}}$ to $\left(\mathcal{P}(\overline{B(x, R)}), d_{2}^{W}\right)$ for all $R>0$ (instead of the convergence of $\left(\overline{B\left(\delta_{x_{i}}, R\right)}, d_{2}^{W}\right)$ to $\left.\left(\overline{B\left(\delta_{x}, R\right)}, d_{2}^{W}\right)\right)$.

The following inspiring precompactness theorem is established by Gromov [Gr, Section 5.A] for the Gromov-Hausdorff convergence, and extended by Fukaya [Fu] to the measured case.

Theorem 5.5 (Gromov-Fukaya precompactness) Let $\left\{\left(M_{i}, g_{i}, \operatorname{vol}_{g_{i}}, x_{i}\right)\right\}_{i \in \mathbb{N}}$ be a sequence of pointed Riemannian manifolds such that

$$
\operatorname{Ric}_{g_{i}} \geq K, \quad \operatorname{dim} M_{i} \leq N
$$

uniformly hold for some $K \in \mathbb{R}$ and $N \in \mathbb{N}$. Then it contains a subsequence which is convergent to some pointed proper metric measure space $(X, d, m, x)$ in the sense of the pointed measured Gromov-Hausdorff convergence.

To be more precise, we choose a complete space as the limit, and then the properness follows from our hypotheses Ric $\geq K$ and $\operatorname{dim} \leq N$. The key ingredient of the proof is the Bishop-Gromov volume comparison (Theorem 3.2 ) from which we derive an upper bound of the doubling constant $\sup _{x \in M, r \leq R} \operatorname{vol}_{g}(B(x, 2 r)) / \operatorname{vol}_{g}(B(x, r))$ for each $R \in(0, \infty)$.

By virtue of Theorem 5.5, starting from a sequence of Riemannian manifolds with a uniform lower Ricci curvature bound, we find the limit space of some subsequence. Such a limit space is not a manifold any more, but should have some inherited properties. The stability of the curvature-dimension condition ensures that we can use it for the investigation of these limit spaces.

We remark that the measures of balls $\operatorname{vol}_{g_{i}}\left(B\left(x_{i}, R\right)\right)$ for $i \in \mathbb{N}$ have a uniform upper bound depending only on $K, N$ and $R$ thanks to Theorem 3.2 . However, it could tend to zero, and then we can not obtain any information on $(X, d, m)$. Therefore we should take a scaling $c_{i} \operatorname{vol}_{g_{i}}$ with some appropriate constant $c_{i}>1$. It does not change anything 
because the weighted Ricci curvature is invariant under scalings of the measure (the weight function of $\tilde{m}=\mathrm{cm}$ is $\psi_{\tilde{m}}=\psi_{m}-\log c$ ). By the same reasoning, it is natural to assume that any bounded open ball has a finite positive measure in the next theorem (see also Remark $5.2(\mathrm{a}))$.

Theorem 5.6 (Stability) Assume that a sequence of pointed proper metric measure spaces $\left\{\left(X_{i}, d_{i}, m_{i}, x_{i}\right)\right\}_{i \in \mathbb{N}}$ uniformly satisfies $\operatorname{CD}(K, N)$ for some $K \in \mathbb{R}$ and $N \in(1, \infty]$ and that it converges to a pointed proper metric measure space $(X, d, m, x)$ in the sense of the pointed measured Gromov-Hausdorff convergence. If, moreover, $0<m(B(x, r))<\infty$ holds for all $x \in X$ and $r \in(0, \infty)$, then $(X, d, m)$ satisfies $\operatorname{CD}(K, N)$.

Outline of proof. The proof of stability goes as follows (along the lines of [LV2], [LV1]). First of all, as we consider only measures with bounded (and hence compact) support in Definition 5.1, we can restrict ourselves to measures with continuous density and compact support. Indeed, it implies by approximation the general case ([LV2, Proposition 3.21, Lemma 3.24], see also Remark 5.2(e)). Given continuous measures $\mu=\rho m, \nu=\sigma m \in$ $\mathcal{P}_{c}^{\text {ac }}(X, m)$ and $\varepsilon_{i}$-approximating maps $\varphi_{i}: X_{i} \longrightarrow X$ as in Definition 5.3, we consider

$$
\mu_{i}=\frac{\rho \circ \varphi_{i}}{\int_{X_{i}} \rho \circ \varphi_{i} d m_{i}} \cdot m_{i}, \quad \nu_{i}=\frac{\sigma \circ \varphi_{i}}{\int_{X_{i}} \sigma \circ \varphi_{i} d m_{i}} \cdot m_{i} \in \mathcal{P}_{c}^{\mathrm{ac}}\left(X_{i}, m_{i}\right)
$$

and take a dynamical optimal transference plan $\Pi_{i} \in \mathcal{P}\left(\Gamma\left(X_{i}\right)\right)$ from $\mu_{i}$ to $\nu_{i}$ satisfying (5.4). Note that $\left(\varphi_{i}\right)_{\sharp} \mu_{i}$ and $\left(\varphi_{i}\right)_{\sharp} \nu_{i}$ weakly converge to $\mu$ and $\nu$, respectively, thanks to the continuity of $\rho$ and $\sigma$.

By a compactness argument ([LV1, Theorem A.45]), extracting a subsequence if necessary, $\Pi_{i}$ converges to some dynamical transference plan $\Pi \in \mathcal{P}(\Gamma(X))$ from $\mu$ to $\nu$ such that, setting

$$
\alpha_{i}(t):=\left(e_{t}\right)_{\sharp} \Pi_{i}, \quad \alpha(t):=\left(e_{t}\right)_{\sharp} \Pi, \quad \pi_{i}:=\left(e_{0} \times e_{1}\right)_{\sharp} \Pi_{i}, \quad \pi:=\left(e_{0} \times e_{1}\right)_{\sharp} \Pi,
$$

$\left(\varphi_{i}\right)_{\sharp} \alpha_{i}$ and $\left(\varphi_{i} \times \varphi_{i}\right)_{\sharp} \pi_{i}$ weakly converge to $\alpha$ and $\pi$, respectively. Then it follows from Proposition 5.4 that $\alpha$ is a minimal geodesic from $\mu$ to $\nu$ and that $\pi$ is an optimal coupling of $\mu$ and $\nu$.

On the one hand, the right-hand side of (5.4) for $\pi_{i}$ converges to that for $\pi$ by virtue of the continuous densities. On the other hand, the monotonicity

$$
U_{\left(\varphi_{i}\right)_{\sharp} m_{i}}\left(\left(\varphi_{i}\right)_{\sharp}\left[\alpha_{i}(t)\right]\right) \leq U_{m_{i}}\left(\alpha_{i}(t)\right)
$$

and the lower semi-continuity

$$
U_{m}(\alpha(t)) \leq \liminf _{i \rightarrow \infty} U_{\left(\varphi_{i}\right)_{\sharp} m_{i}}\left(\left(\varphi_{i}\right)_{\sharp}\left[\alpha_{i}(t)\right]\right)
$$

hold true in general ([LV2, Theorem B.33]). Therefore we obtain (5.4) for $\Pi$ and complete the proof.

Further Reading The definition of the curvature-dimension condition is much indebted to McCann's influential work [Mc1] introducing the important class of functions $\mathcal{D C}_{N}$ as 
well as the displacement convexity along geodesics in the Wasserstein space (see Further Reading in Section 44). Otto and Villani's work [OV] on the relation between such convexity of the entropy and several functional inequalities was also inspiring.

The term 'curvature-dimension condition' is used by Sturm [St3], St4] (and also in [Vi2]) following Bakry and Émery's celebrated work [BE]. Sturm's condition requires that (5.3) is satisfied for all absolutely continuous measures and $U=S_{N^{\prime}}$ for all $N^{\prime} \in[N, \infty]$. Lott and Villani [LV2, [LV1], independently of Sturm, introduced the condition as in Definition 4.6 and call it $N$-Ricci curvature bounded from below by $K$. These conditions are equivalent in non-branching spaces (see Remark 5.2(e) and Subsection 8.1). In locally compact non-branching spaces, it is also possible to extend (5.3) from compactly supported measures to not necessarily compactly supported measures (see [FV]).

See [Fu], Gr, Chapter 3, Section 5.A] and [BBI, Chapters 7, 8] for the basics of (measured) Gromov-Hausdorff convergence and for precompactness theorems. The stability under the measured Gromov-Hausdorff convergence we presented above is due to Lott and Villani [LV2], [LV1]. Sturm [St3], [St4] also proved the stability with respect to a different, his own notion of convegence induced from his $\mathbf{D}$-distance between metric measure spaces. Roughly speaking, the $\mathbf{D}$-distance takes couplings not only for measures, but also for distances (see [St3] for more details).

We also refer to celebrated work of Cheeger and Colding [CC] (mentioned in Further Reading of Section 3) for related geometric approach toward the investigation of limit spaces of Riemannian manifolds of Ricci curvature bounded below. Their strategy is to fully use the fact that it is the limit of Riemannian manifolds. They reveal the detailed local structure of such limit spaces, however, it also turns out that the limit spaces can have highly wild structures (see a survey [We] and the references therein). We can not directly extend Cheeger and Colding's theory to metric measure spaces with the curvature-dimension condition. Their key tool is the Cheeger-Gromoll type splitting theorem, but Banach spaces prevent us to apply it under the curvature-dimension condition (see Subsection 6.3(C) for more details).

\section{Geometric applications}

Metric measure spaces satisfying the curvature-dimension condition $\mathrm{CD}(K, N)$ enjoy many properties common to 'spaces of dimension $\leq N$ and Ricci curvature $\geq K$ '. To be more precise, though $N \in(1, \infty]$ is not necessarily an integer, we will obtain estimates numerically extended to non-integer $N$. Proofs based on optimal transport theory themselves are interesting and inspiring. Although we concentrate on geometric applications in this article, there are also many analytic applications including the Talagrand inequality, logarithmic Sobolev inequality (and hence the normal concentration of measures), global Poincaré inequality and so forth (see [LV2], [LV1]).

\subsection{Generalized Brunn-Minkowski inequality and applications}

Our first application is a generalization of the Brunn-Minkowski inequality (4.2), (4.4) to curved spaces. This follows from the curvature-dimension condition (5.4) applied to $S_{N}$ 
and $\operatorname{Ent}_{m}$ (i.e., (4.11) and (4.12) ) between uniform distributions on two measurable sets. In the particular case of $K=0$, we obtain the concavity of $m^{1 / N}$ or $\log m$ as in (4.2), (4.4). Given two sets $A, B \subset X$ and $t \in(0,1)$, we denote by $Z_{t}(A, B)$ the set of points $\gamma(t)$ such that $\gamma:[0,1] \longrightarrow X$ is a minimal geodesic with $\gamma(0) \in A$ and $\gamma(1) \in B$. We remark that $Z_{t}(A, B)$ is not necessarily measurable regardless the measurability of $A$ and $B$, however, it is not a problem because $m$ is regular (see Remark 4.2).

The following theorem is essentially contained in von Renesse and Sturm [vRS for $N=\infty$, and due to Sturm [St4] for $N<\infty$. Again we will be implicitly indebted to Theorem 6.5 that guarantees that $\operatorname{diam} X \leq \pi \sqrt{(N-1) / K}$ if $K>0$ and $N<\infty$ (see Remark 5.2(b)). Figure 8 represents rough image of the theorem, $Z_{1 / 2}(A, B)$ has more measure in a positively curved space, and less measure in a negatively curved space (compare this with Figure 1).

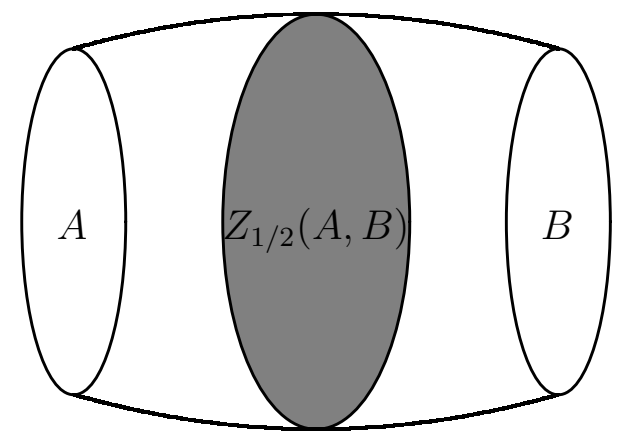

Ric $>0$

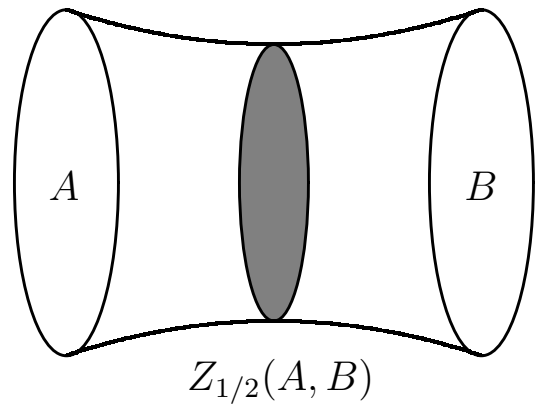

Ric $<0$

Figure 8

Theorem 6.1 (Generalized Brunn-Minkowski inequality) Take a metric measure space $(X, d, m)$ satisfying $\mathrm{CD}(K, N)$ and two measurable sets $A, B \subset X$.

(i) If $N \in(1, \infty)$, then we have

$$
\begin{aligned}
m\left(Z_{t}(A, B)\right)^{1 / N} \geq & (1-t) \inf _{x \in A, y \in B} \beta_{K, N}^{1-t}(d(x, y))^{1 / N} \cdot m(A)^{1 / N} \\
& +t \inf _{x \in A, y \in B} \beta_{K, N}^{t}(d(x, y))^{1 / N} \cdot m(B)^{1 / N}
\end{aligned}
$$

for all $t \in(0,1)$.

(ii) If $N=\infty$ and $0<m(A), m(B)<\infty$, then we have

$$
\begin{aligned}
& \log m\left(Z_{t}(A, B)\right) \\
& \geq(1-t) \log m(A)+t \log m(B)+\frac{K}{2}(1-t) t d_{2}^{W}\left(\frac{\chi_{A}}{m(A)} m, \frac{\chi_{B}}{m(B)} m\right)^{2}
\end{aligned}
$$

for all $t \in(0,1)$. 
Proof. Similarly to Theorem 4.1, we can assume that $A$ and $B$ are bounded and of positive measure. Set

$$
\mu_{0}:=\frac{\chi_{A}}{m(A)} \cdot m, \quad \mu_{1}:=\frac{\chi_{B}}{m(B)} \cdot m, \quad \hat{\beta}^{t}:=\inf _{x \in A, y \in B} \beta_{K, N}^{t}(d(x, y)) .
$$

(i) We consider $U(r)=N r\left(1-r^{-1 / N}\right)$ and recall from (5.1) that, for $\mu=\rho m+\mu^{s}$,

$$
U_{m}(\mu)=N\left(1-\int_{X} \rho^{1-1 / N} d m\right)
$$

Hence it follows from $\operatorname{CD}(K, N)$ that there is a minimal geodesic $\alpha:[0,1] \longrightarrow \mathcal{P}(X)$ from $\mu_{0}$ to $\mu_{1}$ as well as an optimal coupling $\pi$ such that, for all $t \in(0,1)$,

$$
\begin{aligned}
-\int_{X} \rho_{t}^{1-1 / N} d m \leq & -(1-t) \int_{X \times X}\left\{m(A) \beta_{K, N}^{1-t}(d(x, y))\right\}^{1 / N} d \pi(x, y) \\
& -t \int_{X \times X}\left\{m(B) \beta_{K, N}^{t}(d(x, y))\right\}^{1 / N} d \pi(x, y) \\
\leq & -(1-t)\left(\hat{\beta}^{1-t}\right)^{1 / N} m(A)^{1 / N}-t\left(\hat{\beta}^{t}\right)^{1 / N} m(B)^{1 / N}
\end{aligned}
$$

where we set $\alpha(t)=\rho_{t} m+\mu_{t}^{s}$. Then the Hölder inequality yields

$$
\int_{X} \rho_{t}^{-1 / N} \cdot \rho_{t} d m \leq\left(\int_{\operatorname{supp} \rho_{t}} \rho_{t}^{-1} \cdot \rho_{t} d m\right)^{1 / N}=m\left(\operatorname{supp} \rho_{t}\right)^{1 / N} \leq m\left(Z_{t}(A, B)\right)^{1 / N} .
$$

This completes the proof for $N<\infty$.

(ii) We argue similarly and obtain from (4.12) that

$$
\operatorname{Ent}_{m}(\alpha(t)) \leq-(1-t) \log m(A)-t \log m(B)-\frac{K}{2}(1-t) t d_{2}^{W}\left(\mu_{0}, \mu_{1}\right)^{2} .
$$

Note that, since $\operatorname{Ent}_{m}(\alpha(t))<\infty, \alpha(t)$ is absolutely continuous and written as $\alpha(t)=\rho_{t} m$. Furthermore, Jensen's inequality applied to the convex function $s \longmapsto s \log s$ shows

$$
\begin{aligned}
\operatorname{Ent}(\alpha(t)) & =m\left(\operatorname{supp} \rho_{t}\right) \int_{\operatorname{supp} \rho_{t}} \rho_{t} \log \rho_{t} \frac{d m}{m\left(\operatorname{supp} \rho_{t}\right)} \\
& \geq m\left(\operatorname{supp} \rho_{t}\right) \int_{\operatorname{supp} \rho_{t}} \rho_{t} \frac{d m}{m\left(\operatorname{supp} \rho_{t}\right)} \cdot \log \left(\int_{\operatorname{supp} \rho_{t}} \rho_{t} \frac{d m}{m\left(\operatorname{supp} \rho_{t}\right)}\right) \\
& =-\log m\left(\operatorname{supp} \rho_{t}\right) \geq-\log m\left(Z_{t}(A, B)\right) .
\end{aligned}
$$

We complete the proof.

As a corollary, we find that $m$ has no atom unless $X$ consists of a single point.

Corollary 6.2 If $(X, d, m)$ contains more than two points and if it satisfies $\operatorname{CD}(K, N)$ with some $K \in \mathbb{R}$ and $N \in(1, \infty]$, then any one point set $\{x\} \subset X$ has null measure. 
Proof. It is sufficient to show the case of $N=\infty$ (due to Remark 5.2(c)). Put $A=\{x\}$ and assume that $m(\{x\})>0$ holds. Take $r>0$ with $m(B(x, 2 r) \backslash B(x, r))>0$ (it is the case for small $r>0)$, and note that

$$
\left.Z_{t}(\{x\}, B(x, 2 r) \backslash B(x, r))\right) \subset B(x, 2 t r) \backslash B(x, t r)
$$

for all $t \in(0,1)$. Thus we apply Theorem 6.1 (ii) with $t=2^{-k}, k \in \mathbb{N}$, and find

$$
\begin{aligned}
& \log m\left(B\left(x, 2^{1-k} r\right) \backslash B\left(x, 2^{-k} r\right)\right) \\
& \geq\left(1-2^{-k}\right) \log (m(\{x\}))+2^{-k} \log (m(B(x, 2 r) \backslash B(x, r)))-\frac{|K|}{2}\left(1-2^{-k}\right) 2^{-k}(2 r)^{2} .
\end{aligned}
$$

Summing this up in $k \in \mathbb{N}$, we observe

$$
\sum_{k=1}^{\infty} \log m\left(B\left(x, 2^{1-k} r\right) \backslash B\left(x, 2^{-k} r\right)\right)=\infty .
$$

This is a contradiction since we have

$$
\begin{aligned}
\sum_{k=1}^{\infty} \log m\left(B\left(x, 2^{1-k} r\right) \backslash B\left(x, 2^{-k} r\right)\right) & \leq \sum_{k=1}^{\infty} m\left(B\left(x, 2^{1-k} r\right) \backslash B\left(x, 2^{-k} r\right)\right) \\
& =m(B(x, 2 r) \backslash\{x\})<\infty
\end{aligned}
$$

Under $\operatorname{CD}(K, N)$ of the finite dimension $N<\infty$, applying Theorem 6.1(i) to thin annuli shows a generalization of the Bishop-Gromov volume comparison theorem (Theorem 3.2, see also (4.7)).

Theorem 6.3 (Generalized Bishop-Gromov volume comparison) Suppose that a metric measure space $(X, d, m)$ satisfies $\operatorname{CD}(K, N)$ with $K \in \mathbb{R}$ and $N \in(1, \infty)$. Then we have

$$
\frac{m(B(x, R))}{m(B(x, r))} \leq \frac{\int_{0}^{R} \mathbf{s}_{K, N}(t)^{N-1} d t}{\int_{0}^{r} \mathbf{s}_{K, N}(t)^{N-1} d t}
$$

for all $x \in X$ and $0<r<R(\leq \pi \sqrt{(N-1) / K}$ if $K>0)$.

Proof. The proof is essentially the same as the Riemannian case. We apply Theorem 6.1(i) to concentric thin annuli and obtain an estimate corresponding to the Bishop area comparison of concentric spheres (3.4). Then we take the sum and the limit (instead of integration), and obtain the theorem.

We give more detailed calculation for thoroughness. For any annulus $B\left(x, r_{2}\right) \backslash B\left(x, r_{1}\right)$ and $t \in(0,1)$, Theorem 6.1(i) and Corollary 6.2 yield that

$$
\begin{aligned}
m\left(B\left(x, t r_{2}\right) \backslash B\left(x, t r_{1}\right)\right) & \geq t^{N} \inf _{d \in\left[r_{1}, r_{2}\right]}\left(\frac{\mathbf{s}_{K, N}(t d)}{t \mathbf{s}_{K, N}(d)}\right)^{N-1} m\left(B\left(x, r_{2}\right) \backslash B\left(x, r_{1}\right)\right) \\
& \geq t \cdot \frac{\inf _{d \in\left[r_{1}, r_{2}\right]} \mathbf{s}_{K, N}(t d)^{N-1}}{\sup _{d \in\left[r_{1}, r_{2}\right]} \mathbf{s}_{K, N}(d)^{N-1}} m\left(B\left(x, r_{2}\right) \backslash B\left(x, r_{1}\right)\right) .
\end{aligned}
$$


This corresponds to (3.4) in the Riemannian case. Set $h(t):=\mathbf{s}_{K, N}(t)^{N-1}$ for brevity, and put $t_{L}:=(r / R)^{1 / L}<1$ for $L \in \mathbb{N}$. Applying (6.1) to $r_{1}=t_{L} r, r_{2}=r$ and $t=t_{L}^{l-1}$ for $l \in \mathbb{N}$, we have

$$
\begin{aligned}
m(B(x, r)) & =\sum_{l=1}^{\infty} m\left(B\left(x, t_{L}^{l-1} r\right) \backslash B\left(x, t_{L}^{l} r\right)\right) \\
& \geq\left\{\sum_{l=1}^{\infty} t_{L}^{l-1} \frac{\inf _{d \in\left[t_{L} r, r\right]} h\left(t_{L}^{l-1} d\right)}{\sup _{d \in\left[t_{L} r, r\right]} h(d)}\right\} m\left(B(x, r) \backslash B\left(x, t_{L} r\right)\right) .
\end{aligned}
$$

We similarly deduce from (6.1) with $r_{1}=t_{L}^{l-L} r, r_{2}=t_{L}^{l-1-L} r$ and $t=t_{L}^{L-l+1}$ for $l=$ $1, \ldots, L$ that

$$
\begin{aligned}
& m\left(B(x, r) \backslash B\left(x, t_{L} r\right)\right) \sum_{l=1}^{L} t_{L}^{l-1} \sup _{d \in\left[t_{L}^{l-L} r t_{L}^{l-1-L} r\right]} h(d) \\
& \geq t_{L}^{L} \inf _{d \in\left[t_{L} r, r\right]} h(d) \sum_{l=1}^{L} m\left(B\left(x, t_{L}^{l-1-L} r\right) \backslash B\left(x, t_{L}^{l-L} r\right)\right) \\
& =\frac{r}{R} \inf _{d \in\left[t_{L} r, r\right]} h(d) \cdot m(B(x, R) \backslash B(x, r)) .
\end{aligned}
$$

Combining these, we obtain

$$
\begin{aligned}
& m(B(x, r)) \cdot \sum_{l=1}^{L}\left(t_{L}^{l-1}-t_{L}^{l}\right) R \sup _{d \in\left[t_{L} R, R\right]} h\left(t_{L}^{l-1} d\right) \\
& =\left(1-t_{L}\right) R \cdot m(B(x, r)) \cdot \sum_{l=1}^{L} t_{L}^{l-1} \sup _{d \in\left[t_{L}^{l-L} r, t_{L}^{l-1-L} r\right]} h(d) \\
& \geq\left(1-t_{L}\right) R \cdot\left\{\sum_{l=1}^{\infty} t_{L}^{l-1} \frac{\inf _{d \in\left[t_{L} r, r\right]} h\left(t_{L}^{l-1} d\right)}{\sup _{d \in\left[t_{L} r, r\right]} h(d)}\right\} \cdot \frac{r}{R} \inf _{d \in\left[t_{L} r, r\right]} h(d) \cdot m(B(x, R) \backslash B(x, r)) \\
& \geq m(B(x, R) \backslash B(x, r)) \cdot \frac{\inf _{d \in\left[t_{L} r, r\right]} h(d)}{\sup _{d \in\left[t_{L} r, r\right]} h(d)} \sum_{l=1}^{\infty}\left(t_{L}^{l-1}-t_{L}^{l}\right) r \inf _{d \in\left[t_{L} r, r\right]} h\left(t_{L}^{l-1} d\right) .
\end{aligned}
$$

Letting $L$ diverge to infinity shows

$$
m(B(x, r)) \int_{r}^{R} \mathbf{s}_{K, N}(t)^{N-1} d t \geq m(B(x, R) \backslash B(x, r)) \int_{0}^{r} \mathbf{s}_{K, N}(t)^{N-1} d t .
$$

This corresponds to (3.5) in the Riemannian case, and the same calculation as the last step of the proof of Theorem 3.2 completes the proof.

Theorem 6.3 shows that the doubling constant $\sup _{x \in X, r \leq R} m(B(x, 2 r)) / m(B(x, r))$ is bounded for each $R \in(0, \infty)$, therefore $X$ is proper (see also the paragraph following Theorem 5.5).

Corollary 6.4 Assume that $(X, d, m)$ satisfies $\operatorname{CD}(K, N)$ for some $K \in \mathbb{R}$ and $N \in$ $(1, \infty)$. Then $(X, d)$ is proper. 
Next we generalize the Bonnet-Myers diameter bound (Corollary 3.4). We remark that the proof below uses Theorem 6.1(i) only for pairs of a point $A=\{x\}$ and a set $B \subset B(x, \pi \sqrt{(N-1) / K})$, so that it is consistent with Remark 5.2(b). The following proof is due to Oh1].

Theorem 6.5 (Generalized Bonnet-Myers diameter bound) Suppose that a metric measure space $(X, d, m)$ satisfies $\operatorname{CD}(K, N)$ with $K>0$ and $N \in(1, \infty)$. Then we have the following:

(i) It holds that $\operatorname{diam} X \leq \pi \sqrt{(N-1) / K}$.

(ii) Each $x \in X$ has at most one point of distance $\pi \sqrt{(N-1) / K}$ from $x$.

Proof. It is enough to consider the case $K=N-1$ thanks to the scaling property Remark 5.2 (a).

(i) Suppose that there is a pair of points $x, y \in X$ with $d(x, y)>\pi$, set $\delta:=d(x, y)-$ $\pi>0$ and take a minimal geodesic $\gamma:[0, \pi+\delta] \longrightarrow X$ from $x$ to $y$. Choosing a different point on $\gamma$ if necessary, we can assume $\delta<\pi / 2$. For $\varepsilon \in(0, \delta)$, we apply Theorem 6.1(i) between $\{\gamma(\delta+\varepsilon)\}$ and $B(y, \varepsilon)$ with $t=(\pi-\delta-\varepsilon) / \pi$ and obtain

$$
\frac{m\left(Z_{t}(\{\gamma(\delta+\varepsilon)\}, B(y, \varepsilon))\right)}{m(B(y, \varepsilon))} \geq t^{N} \inf _{r \in(\pi-2 \varepsilon, \pi)}\left(\frac{\sin (t r)}{t \sin r}\right)^{N-1}=t\left(\frac{\sin (t(\pi-2 \varepsilon))}{\sin (\pi-2 \varepsilon)}\right)^{N-1} .
$$

Then it follows from $t(\pi-2 \varepsilon) \leq t \pi=\pi-\delta-\varepsilon$ that

$$
\frac{m\left(Z_{t}(\{\gamma(\delta+\varepsilon)\}, B(y, \varepsilon))\right)}{m(B(y, \varepsilon))} \geq \frac{\pi-\delta-\varepsilon}{\pi}\left(\frac{\sin (\delta+\varepsilon)}{\sin 2 \varepsilon}\right)^{N-1} \rightarrow \infty
$$

as $\varepsilon$ tends to zero. Given $z \in B(y, \varepsilon)$, we take a minimal geodesic $\eta:[0,1] \longrightarrow X$ from $\gamma(\delta+\varepsilon)$ to $z$ (see Figure 9 ), and derive from the triangle inequality that

$$
d(\gamma(\delta+\varepsilon), \eta(t))=t d(\gamma(\delta+\varepsilon), z)<t\{d(\gamma(\delta+\varepsilon), y)+\varepsilon\}=\pi-\delta-\varepsilon .
$$

Moreover, we deduce from $d(\gamma(\delta+\varepsilon), z)<\pi$ that

$$
\begin{aligned}
d(x, \eta(t)) & \geq d(x, z)-d(z, \eta(t))>\pi+\delta-\varepsilon-(1-t) d(\gamma(\delta+\varepsilon), z) \\
& >\pi+\delta-\varepsilon-(1-t) \pi=\pi-2 \varepsilon .
\end{aligned}
$$

Thus we have

$$
\begin{aligned}
Z_{t}(\{\gamma(\delta+\varepsilon)\}, B(y, \varepsilon)) & \subset B(\gamma(\delta+\varepsilon), \pi-\delta-\varepsilon) \backslash B(x, \pi-2 \varepsilon) \\
& \subset B(x, \pi) \backslash B(x, \pi-2 \varepsilon) .
\end{aligned}
$$

Combining this with (6.3), we conclude

$$
\lim _{\varepsilon \downarrow 0} \frac{m(B(x, \pi) \backslash B(x, \pi-2 \varepsilon))}{m(B(y, \varepsilon))}=\infty .
$$




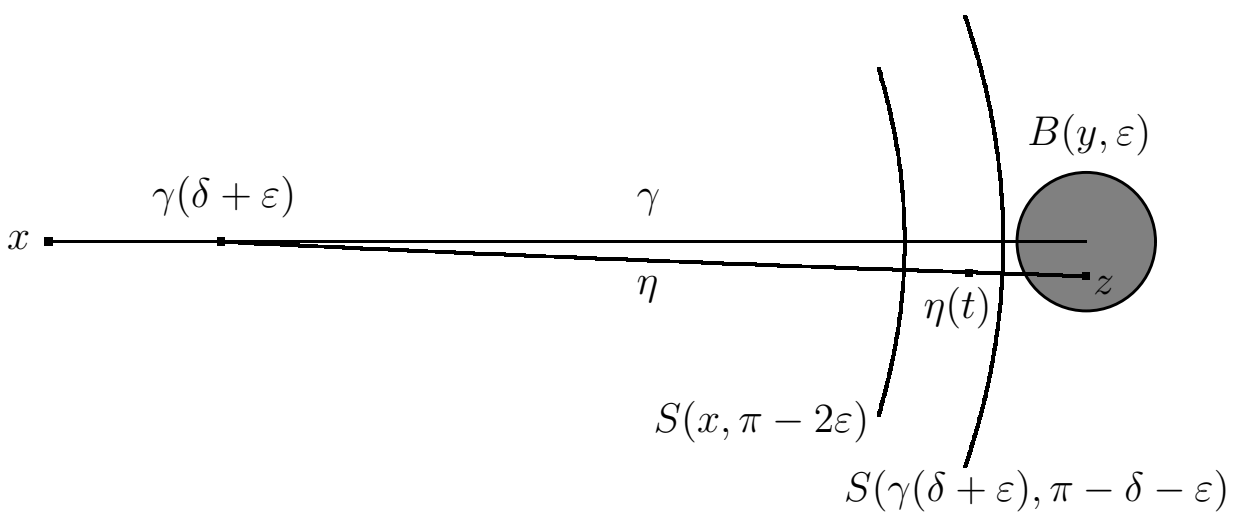

Figure 9

Furthermore, (6.2) and Theorem 6.3 show that

$$
\begin{aligned}
& m(B(x, \pi) \backslash B(x, \pi-2 \varepsilon)) \leq \frac{\int_{\pi-2 \varepsilon}^{\pi} \sin ^{N-1} r d r}{\int_{0}^{\pi-2 \varepsilon} \sin ^{N-1} r d r} m(B(x, \pi-2 \varepsilon)) \\
& =\frac{\int_{0}^{2 \varepsilon} \sin ^{N-1} r d r}{\int_{0}^{\pi-2 \varepsilon} \sin ^{N-1} r d r} m(B(x, \pi-2 \varepsilon)) \leq m(B(x, 2 \varepsilon)) .
\end{aligned}
$$

Hence we have, again due to Theorem 6.3 (with $K=0$ ),

$$
m(B(x, \pi) \backslash B(x, \pi-2 \varepsilon)) \leq m(B(x, 2 \varepsilon)) \leq 2^{N} m(B(x, \varepsilon)) .
$$

Therefore we obtain $\lim _{\varepsilon \downarrow 0} m(B(x, \varepsilon)) / m(B(y, \varepsilon))=\infty$. This is a contradiction because we can exchange the roles of $x$ and $y$.

(ii) We first see that $m(S(x, \pi))=0$, where $S(x, \pi):=\{y \in X \mid d(x, y)=\pi\}$. Given small $\varepsilon>0$, take $\left\{x_{i}\right\}_{i=1}^{k} \subset S(x, 2 \varepsilon)$ such that $S(x, 2 \varepsilon) \subset \bigcup_{i=1}^{k} B\left(x_{i}, 2 \varepsilon\right)$ and $d\left(x_{i}, x_{j}\right) \geq 2 \varepsilon$ holds if $i \neq j$. Then, for any $y \in S(x, \pi)$, there is some $x_{i}$ so that $d\left(y, x_{i}\right)<(\pi-2 \varepsilon)+2 \varepsilon=$ $\pi$, while $d\left(y, x_{i}\right) \geq \pi-2 \varepsilon$ holds in general. Thus we have

$$
\begin{aligned}
m(S(x, \pi)) & \leq m\left(\bigcup_{i=1}^{k} B\left(x_{i}, \pi\right) \backslash B\left(x_{i}, \pi-2 \varepsilon\right)\right) \\
& \leq \sum_{i=1}^{k} m\left(B\left(x_{i}, \pi\right) \backslash B\left(x_{i}, \pi-2 \varepsilon\right)\right) .
\end{aligned}
$$

Then it follows from (6.4) that

$$
\begin{aligned}
m(S(x, \pi)) & \leq \sum_{i=1}^{k} m\left(B\left(x_{i}, 2 \varepsilon\right)\right) \leq 2^{N} \sum_{i=1}^{k} m\left(B\left(x_{i}, \varepsilon\right)\right) \\
& =2^{N} m\left(\bigcup_{i=1}^{k} B\left(x_{i}, \varepsilon\right)\right) \leq 2^{N} m(B(x, 3 \varepsilon)) .
\end{aligned}
$$


Letting $\varepsilon$ go to zero shows $m(S(x, \pi))=0$.

Now we suppose that there are two mutually distinct points $y, z \in X$ such that $d(x, y)=d(x, z)=\pi$. On the one hand, we derive from (6.4) that

$$
m(B(x, r)) \geq m(B(x, \pi) \backslash B(x, \pi-r))
$$

for $r \in(0, \pi / 2)$. On the other hand, as $B(y, r) \subset X \backslash B(x, \pi-r)$ and $m(S(x, \pi))=0$, we find

$$
m(B(y, r)) \leq m(B(x, \pi) \backslash B(x, \pi-r)) .
$$

Hence we obtain $m(B(x, r)) \geq m(B(y, r))$ and similarly $m(B(y, r)) \geq m(B(x, r))$. This implies

$$
m(B(x, r))=m(B(y, r))=m(B(z, r))=m(B(x, \pi) \backslash B(x, \pi-r)) .
$$

Then we have, for $\varepsilon<d(y, z) / 2$,

$$
\begin{aligned}
2 m(B(x, \varepsilon)) & =m(B(y, \varepsilon))+m(B(z, \varepsilon))=m(B(y, \varepsilon) \cup B(z, \varepsilon)) \\
\leq m(B(x, \pi) \backslash B(x, \pi-\varepsilon)) & =m(B(x, \varepsilon)) .
\end{aligned}
$$

This is obviously a contradiction.

For $(X, d, m)$ satisfying $\operatorname{CD}(K, \infty)$ with $K>0$, though $X$ is not necessarily bounded (see Example 4.5), we can verify that $m(X)$ is finite ([St3, Theorem 4.26]).

\subsection{Maximal diameter}

In Riemannian geometry, it is well known that the maximal diameter $\pi$ among (unweighted) Riemannian manifolds of Ricci curvature $\geq n-1$ is achieved only by the unit sphere $\mathbb{S}^{n}$. In our case, however, orbifolds $\mathbb{S}^{n} / \Gamma$ can also have the maximal diameter. Hence what we can expect is a decomposition into a spherical suspension (in some sense). Due to the scaling property (Remark $[5.2($ a) ), we consider only the case of $K=N-1>0$. See [Oh2] for more precise discussion of the following theorem, and Subsection 8.1 for the definition of the non-branching property.

Theorem 6.6 Assume that $(X, d, m)$ is non-branching and satisfies $\operatorname{CD}(N-1, N)$ for some $N \in(1, \infty)$ as well as $\operatorname{diam} X=\pi$. Then $(X, m)$ is the spherical suspension of some topological measure space.

Outline of proof. Fix $x_{N}, x_{S} \in X$ with $d\left(x_{N}, x_{S}\right)=\pi$. Then it follows from (6.4) that

$$
m\left(B\left(x_{N}, r\right)\right)+m\left(B\left(x_{S}, \pi-r\right)\right)=m(X)
$$

for all $r \in(0, \pi)$. This together with the non-branching property shows that, for any $z \in X \backslash\left\{x_{N}, x_{S}\right\}$, there exists a unique minimal geodesic from $x_{N}$ to $x_{S}$ passing through $z$. 
Now we introduce the set $Y$ consisting of unit speed minimal geodesics from $x_{N}$ to $x_{S}$, and equip it with the distance

$$
d_{Y}\left(\gamma_{1}, \gamma_{2}\right):=\sup _{0 \leq t \leq \pi} d_{X}\left(\gamma_{1}(t), \gamma_{2}(t)\right)
$$

We consider $S Y:=(Y \times[0, \pi]) / \sim$, where $\left(\gamma_{1}, t_{1}\right) \sim\left(\gamma_{2}, t_{2}\right)$ holds if $t_{1}=t_{2}=0$ or $t_{1}=t_{2}=\pi$. We equip $S Y$ with the topology naturally induced from $d_{Y}$. Then the map $\Psi: S Y \ni(\gamma, t) \longmapsto \gamma(t) \in X$ is well-defined and continuous. Define the mesures $\nu$ on $Y$ and $\omega$ on $S Y$ by

$$
\begin{aligned}
\nu(W) & :=\left\{\int_{0}^{\pi} \sin ^{N-1} t d t\right\}^{-1} m(\Psi(W \times[0, \pi])), \\
d \omega & :=d \nu \times\left(\sin ^{N-1} t d t\right) .
\end{aligned}
$$

Then one can prove that $(S Y, \omega)$ is regarded as the spherical suspension of $(Y, \nu)$ as topological measure spaces, and that $\Psi:(S Y, \omega) \longrightarrow(X, m)$ is homeomorphic and measurepreserving. We use the non-branching property for the continuity of $\Psi^{-1}$.

\subsection{Open questions}

We close the section with a list of open questions.

(A) (Beyond Theorem 6.6) There is room for improvement of Theorem 6.6. Is the non-branching property necessary? Can one say anything about the relation between the distances of $S Y$ and $X$ ? Does $\left(Y, d_{Y}, \nu\right)$ satisfy $\operatorname{CD}(N-2, N-1)$ ?

If $X$ is an $n$-dimensional Alexandrov space of curvature $\geq 1$ with $\operatorname{diam} X=\pi$, then it is isometric to the spherical suspension of some $(n-1)$-dimensional Alexandrov space of curvature $\geq 1$. It is generally difficult to derive something about distance from the curvature-dimension condition. We also do not know any counterexample.

(B) (Extremal case of Lichnerowicz inequality) Related to Theorem 6.5, we know the following ([LV1, Theorem 5.34]).

Theorem 6.7 (Generalized Lichnerowicz inequality) Assume that $(X, d, m)$ satisfies $\mathrm{CD}(K, N)$ for some $K>0$ and $N \in(1, \infty)$. Then we have

$$
\int_{X} f^{2} d m \leq \frac{N-1}{K N} \int_{X}\left|\nabla^{-} f\right|^{2} d m
$$

for any Lipschitz function $f: X \longrightarrow \mathbb{R}$ with $\int_{X} f d m=0$.

Here $\left|\nabla^{-} f\right|$ is the generalized gradient of $f$ defined by

$$
\left|\nabla^{-} f\right|(x):=\limsup _{y \rightarrow x} \frac{\max \{f(x)-f(y), 0\}}{d(x, y)} .
$$

The proof is done via careful calculation using (5.4) for $S_{N}$ between $m(X)^{-1} \cdot m$ and its perturbation $(1+\varepsilon f) m(X)^{-1} \cdot m$. The inequality (6.5) means that the lowest positive eigenvalue of the Laplacian is larger than or equal to $K N /(N-1)$. The constant $(N-$ 
$1) / K N$ in (6.5) is sharp. Moreover, in Riemannian geometry, it is known that the best constant with $N=\operatorname{dim} M$ is achieved only by spheres.

In our general setting, it is not known whether the best constant is achieved only by spaces of maximal diameter $\pi \sqrt{(N-1) / K}$. If so, then Theorem 6.6 provides us a decomposition into a spherical suspension (for non-branching spaces).

(C) (Splitting) In Riemannian geometry, Cheeger and Gromoll's [CG] celebrated theorem asserts that, if a complete Riemannian manifold of nonnegative Ricci curvature admits an isometric embedding of the real line $\mathbb{R} \hookrightarrow M$, then $M$ isometrically splits off $\mathbb{R}$, namely $M$ is isometric to a product space $M^{\prime} \times \mathbb{R}$, where $M^{\prime}$ again has the nonnegative Ricci curvature. We can repeat this procedure if $M^{\prime}$ contains a line. This is an extremely deep theorem, and its generalization is a key tool of Cheeger and Colding's seminal work [CC] (see Further Reading of Section 5).

Kuwae and Shioya [KS3] consider (weighted) Alexandrov spaces of curvature $\geq-1$ with nonnegative Ricci curvature in terms of the measure contraction property (see Subsection 8.3). They show that, if such an Alexandrov space contains an isometric copy of the real line, then it splits off $\mathbb{R}$ as topological measure spaces (compare this with Theorem [6.6). This is recently strengthened into an isometric splitting by [ZZ] under a slightly stronger notion of Ricci curvature bound in terms of Petrunin's second variation formula $([$ Pe1 $])$.

For general metric measure spaces satisfying $\mathrm{CD}(0, N)$, the isometric splitting is false because $n$-dimensional Banach spaces satisfy $\operatorname{CD}(0, n)$ (Theorem 7.3) and do not split in general. The homeomorphic, measure-preserving splitting could be true, but it is open even for non-branching spaces.

(D) (Lévy-Gromov isoperimetric inequality) Another challenging problem is to show (some appropriate variant of) the Lévy-Gromov isoperimetric inequality using optimal transport. Most known proofs in the Riemannian case appeal to the deep existence and regularity theory of minimal surfaces which can not be expected in singular spaces.

For instance, let us consider the isoperimetric profile $I_{M}:(0, m(M)) \longrightarrow(0, \infty)$ of a weighted Riemannian manifold $(M, g, m)$ with $m=e^{-\psi} \operatorname{vol}_{g}$, i.e., $I_{M}(V)$ is the least perimeter of sets with volume $V$. Bayle Bay shows that the differential inequality

$$
\left(I_{M}^{N /(N-1)}\right)^{\prime \prime} \leq-\frac{K N}{N-1} I_{M}^{1 /(N-1)-1}
$$

holds if $\operatorname{Ric}_{N} \geq K$, which immediately implies the corresponding Lévy-Gromov isoperimetric inequality

$$
\frac{I_{M}(t \cdot m(M))}{m(M)} \geq I_{K, N}(t)
$$

for $t \in[0,1]$, where $I_{K, N}$ is the isoperimetric profile of the $N$-dimensional space form of constant sectional curvature $K /(N-1)$ equipped with the normalized measure (extended to non-integer $N$ numerically). The concavity estimate (6.6) seems to be related to the Brunn-Minkowski inequality, however, Bayle's proof of $(\underline{6.6})$ is based on the variational formulas of minimal surfaces (see also [Mo, Chapter 18]). More analytic approach could work in metric measure spaces, but we need a new idea for it.

Further Reading The generalized Brunn-Minkowski inequality (Theorem 6.1) is essentially contained in the proof of [vRS, Theorem 1.1] for $N=\infty$, and due to Sturm [St4] 
for $N<\infty$ (also the Brascamp-Lieb inequality in [CMS1 implies it in the unweighted Riemannian situation with $N=n$ ). It is used as a key tool in the proof of the derivation of the Ricci curvature bound from the curvature-dimension condition (see Theorem 4.6). Some more related interpolation inequalities can be found in CMS1], these all were new even for Riemannian manifolds.

The relation between $\operatorname{CD}(K, \infty)$ and functional inequalities such as the Talagrand, logarithmic Sobolev and the global Poincaré inequalities are studied by Otto and Villani [OV] and Lott and Villani [LV2, Section 6]. We refer to [O1], BoS for related work on discrete spaces (see also Subsection [7.3(B)), and to [St2, [Vi2, Chapter 25], OT] for the relation between variants of these functional inequalities and the displacement convexity of generalized entropies. Theorems 6.5 and 6.6 are due to [Oh1] and [Oh2], where the proof is given in terms of the measure contraction property (see Subsection 8.3).

\section{The curvature-dimension condition in Finsler ge- ometry}

In this section, we demonstrate that almost everything so far works well also in the Finsler setting. In fact, the equivalence between $\operatorname{Ric}_{N} \geq K$ and $\operatorname{CD}(K, N)$ is extended by introducing an appropriate notion of the weighted Ricci curvature. Then we explain why this is significant and discuss two potential applications. We refer to [BCS] and [Sh2] for the fundamentals of Finsler geometry, and the main reference of the section is [Oh5].

\subsection{A brief introduction to Finsler geometry}

Let $M$ be an $n$-dimensional connected $C^{\infty}$-manifold. Given a local coordinate $\left(x^{i}\right)_{i=1}^{n}$ on an open set $U \subset M$, we always consider the coordinate $\left(x^{i}, v^{i}\right)_{i=1}^{n}$ on $T U$ given by

$$
v=\left.\sum_{i=1}^{n} v^{i} \frac{\partial}{\partial x^{i}}\right|_{x} \in T_{x} M .
$$

Definition 7.1 (Finsler structures) A $C^{\infty}$-Finsler structure is a nonnegative function $F: T M \longrightarrow[0, \infty)$ satisfying the following three conditions:

(1) (Regularity) $F$ is $C^{\infty}$ on $T M \backslash 0$, where 0 stands for the zero section;

(2) (Positive homogeneity) $F(\lambda v)=\lambda F(v)$ holds for all $v \in T M$ and $\lambda \geq 0$;

(3) (Strong convexity) Given a local coordinate $\left(x^{i}\right)_{i=1}^{n}$ on $U \subset M$, the $n \times n$ matrix

$$
\left(g_{i j}(v)\right)_{i, j=1}^{n}:=\left(\frac{1}{2} \frac{\partial^{2}\left(F^{2}\right)}{\partial v^{i} \partial v^{j}}(v)\right)_{i, j=1}^{n}
$$

is positive-definite for all $v \in T_{x} M \backslash 0, x \in U$. 
In other words, each $\left.F\right|_{T_{x} M}$ is a $C^{\infty}$-Minkowski norm (see Example 7.4(a) below for the precise definition) and it varies $C^{\infty}$-smoothly also in the horizontal direction. We remark that the homogeneity (2) is imposed only in the positive direction, so that $F(-v) \neq$ $F(v)$ is allowed. The positive-definite symmetric matrix $\left(g_{i j}(v)\right)_{i, j=1}^{n}$ in (7.1) defines the Riemannian structure $g_{v}$ on $T_{x} M$ through

$$
g_{v}\left(\left.\sum_{i=1}^{n} v_{1}^{i} \frac{\partial}{\partial x^{i}}\right|_{x},\left.\sum_{j=1}^{n} v_{2}^{j} \frac{\partial}{\partial x^{j}}\right|_{x}\right):=\sum_{i, j=1}^{n} g_{i j}(v) v_{1}^{i} v_{2}^{j} .
$$

Note that $F(v)^{2}=g_{v}(v, v)$. If $F$ is coming from a Riemannian structure, then $g_{v}$ always coincides with the original Riemannian metric. In general, the inner product $g_{v}$ is regarded as the best approximation of $F$ in the direction $v$. More precisely, the unit spheres of $F$ and $g_{v}$ are tangent to each other at $v / F(v)$ up to the second order (that is possible thanks to the strong convexity, see Figure 10).

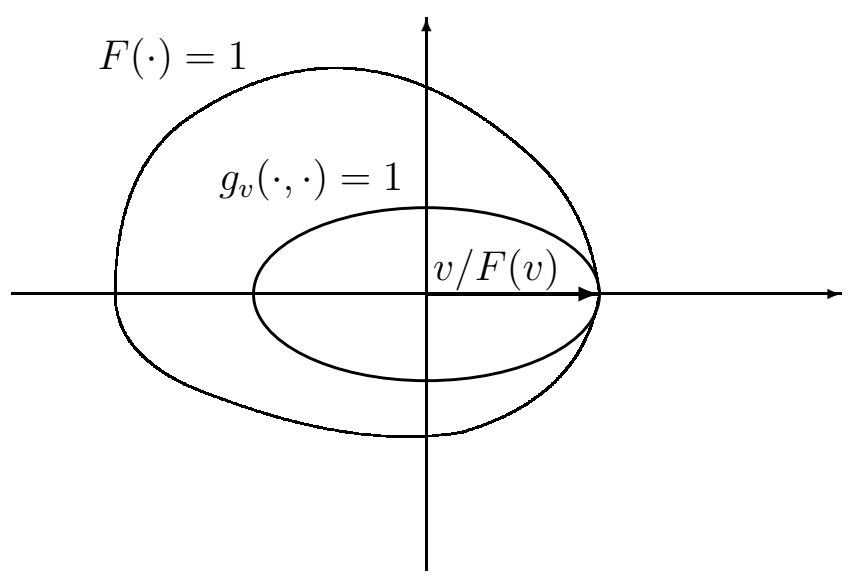

Figure 10

The distance between $x, y \in M$ is naturally defined by

$$
d(x, y):=\inf \left\{\int_{0}^{1} F(\dot{\gamma}) d t \mid \gamma:[0,1] \longrightarrow M, C^{1}, \gamma(0)=x, \gamma(1)=y\right\} .
$$

One remark is that the nonsymmetry $d(x, y) \neq d(y, x)$ may come up as $F$ is only positively homogeneous. Thus it is not totally correct to call $d$ a distance, it might be called cost or action as $F$ is a sort of Lagrangian cost function. Another remark is that the function $d(x, \cdot)^{2}$ is $C^{2}$ at the origin $x$ if and only if $\left.F\right|_{T_{x} M}$ is Riemannian. Indeed, the squared norm $|\cdot|^{2}$ of a Banach (or Minkowski) space $\left(\mathbb{R}^{n},|\cdot|\right)$ is $C^{2}$ at 0 if and only if it is an inner product.

A $C^{\infty}$-curve $\gamma:[0, l] \longrightarrow M$ is called a geodesic if it has constant speed $(F(\dot{\gamma}) \equiv c \in$ $[0, \infty))$ and is locally minimizing (with respect to $d$ ). The reverse curve $\bar{\gamma}(t):=\gamma(l-t)$ is not necessarily a geodesic. We say that $(M, F)$ is forward complete if any geodesic $\gamma:[0, \varepsilon] \longrightarrow M$ is extended to a geodesic $\bar{\gamma}:[0, \infty) \longrightarrow M$. Then any two points $x, y \in M$ are connected by a minimal geodesic from $x$ to $y$. 


\subsection{Weighted Ricci curvature and the curvature-dimension con- dition}

We introduced distance and geodesics in a natural (metric geometric) way, but the definition of curvature is more subtle. The flag and Ricci curvatures on Finsler manifolds, corresponding to the sectional and Ricci curvatures in Riemannian geometry, are defined via some connection as in the Riemannian case. The choice of connection is not unique in the Finsler setting, nevertheless, all connections are known to give rise to the same curvature. In these notes, however, we shall follow Shen's idea [Sh2, Chapter 6] of introducing the flag curvature using vector fields and corresponding Riemannian structures (via (7.2)). This intuitive description is not only geometrically understandable, but also useful and inspiring.

Fix a unit vector $v \in T_{x} M \cap F^{-1}(1)$, and extend it to a $C^{\infty}$-vector field $V$ on an open neighborhood $U$ of $x$ in such a way that every integral curve of $V$ is geodesic. In particular, $V(\gamma(t))=\dot{\gamma}(t)$ along the geodesic $\gamma:(-\varepsilon, \varepsilon) \longrightarrow M$ with $\dot{\gamma}(0)=v$. Using (7.2), we equip $U$ with the Riemannian structure $g_{V}$. Then the flag curvature $\mathcal{K}(v, w)$ of $v$ and a linearly independent vector $w \in T_{x} M$ coincides with the sectional curvature with respect to $g_{V}$ of the 2-plane $v \wedge w$ spanned by $v$ and $w$. Similarly, the Ricci curvature $\operatorname{Ric}(v)$ of $v$ (with respect to $F$ ) coincides with the Ricci curvature of $v$ with respect to $g_{V}$. This contains the fact that $\mathcal{K}(v, w)$ is independent of the choice of the extension $V$ of $v$. We remark that $\mathcal{K}(v, w)$ depends not only on the flag $v \wedge w$, but also on the choice of the flagpole $v$ in the flag $v \wedge w$. In particular, $\mathcal{K}(v, w) \neq \mathcal{K}(w, v)$ may happen.

As for measure, on Finsler manifolds, there is no constructive measure as good as the Riemannian volume measure. Therefore, as the theory of weighted Riemannian manifolds, we equip $(M, F)$ with an arbitrary positive $C^{\infty}$-measure $m$ on $M$. Now, the weighted Ricci curvature is defined as follows ([Oh5]). We extend given a unit vector $v \in T_{x} M$ to a $C^{\infty}$ vector field $V$ on a neighborhood $U \ni x$ such that every integral curve is geodesic (or it is sufficient to consider only the tangent vector field $\dot{\gamma}$ of the geodesic $\gamma:(-\varepsilon, \varepsilon) \longrightarrow M$ with $\dot{\gamma}(0)=v$ ), and decompose $m$ as $m=e^{-\Psi(V)} \operatorname{vol}_{g_{V}}$ on $U$. We remark that the weight $\Psi$ is not a function on $M$, but a function on the unit tangent sphere bundle $S M \subset T M$. For simplicity, we set

$$
\partial_{v} \Psi:=\frac{d(\Psi \circ \dot{\gamma})}{d t}(0), \quad \partial_{v}^{2} \Psi:=\frac{d^{2}(\Psi \circ \dot{\gamma})}{d t^{2}}(0) .
$$

Definition 7.2 (Weighted Ricci curvature of Finsler manifolds) For $N \in[n, \infty]$ and a unit vector $v \in T_{x} M$, we define

(1) $\operatorname{Ric}_{n}(v):= \begin{cases}\operatorname{Ric}(v)+\partial_{v}^{2} \Psi & \text { if } \partial_{v} \Psi=0, \\ -\infty & \text { otherwise }\end{cases}$

(2) $\operatorname{Ric}_{N}(v):=\operatorname{Ric}(v)+\partial_{v}^{2} \Psi-\frac{\left(\partial_{v} \Psi\right)^{2}}{N-n}$ for $N \in(n, \infty)$;

(3) $\operatorname{Ric}_{\infty}(v):=\operatorname{Ric}(v)+\partial_{v}^{2} \Psi$.

In other words, $\operatorname{Ric}_{N}(v)$ of $F$ is $\operatorname{Ric}_{N}(v)$ of $g_{V}$ (recall Definition 4.4), so that this curvature coincides with Ric $_{N}$ in weighted Riemannian manifolds. We remark that the 
quantity $\partial_{v} \Psi$ coincides with Shen's S-curvature (also called the mean covariance or mean tangent curvature, see [Sh1], [Sh2], Sh3]). Therefore bounding $\mathrm{Ric}_{n}$ from below makes sense only when the $\mathbf{S}$-curvature vanishes everywhere. This curvature enables us to extend Theorem 4.6 to the Finsler setting ([Oh5]). Therefore all results in the theory of curvaturedimension condition are applicable to general Finsler manifolds.

Theorem 7.3 A forward complete Finsler manifold $(M, F, m)$ equipped with a positive $C^{\infty}$-measure $m$ satisfies $\mathrm{CD}(K, N)$ for some $K \in \mathbb{R}$ and $N \in[n, \infty]$ if and only if $\operatorname{Ric}_{N}(v) \geq K$ holds for all unit vectors $v \in T M$.

We remark that, in the above theorem, the curvature-dimension condition is appropriately extended to nonsymmetric distances. The proof of Theorem 7.3 follows the same line as the Riemannian case, however, we should be careful about nonsymmetric distance and need some more extra discussion due to the fact that the squared distance function $d(x, \cdot)^{2}$ is only $C^{1}$ at $x$.

We present several examples of Finsler manifolds. The flag and Ricci curvatures are calculated in a number of situations, while the weighted Ricci curvature is still relatively much less investigated.

Example 7.4 (a) (Banach/Minkowski spaces with Lebesgue measures) A Minkowski norm $|\cdot|$ on $\mathbb{R}^{n}$ is a nonsymmetric generalization of usual norms. That is to say, $|\cdot|$ is a nonnegative function on $\mathbb{R}^{n}$ satisfying the positive homogeneity $|\lambda v|=\lambda|v|$ for $v \in \mathbb{R}^{n}$ and $\lambda>0$; the convexity $|v+w| \leq|v|+|w|$ for $v, w \in \mathbb{R}^{n}$; and the positivity $|v|>0$ for $v \neq 0$. Note that the unit ball of $|\cdot|$ is a convex (but not necessarily symmetric to the origin) domain containing the origin in its interior (see Figure 10, where $F$ is a Minkowski norm).

A Banach or Minkowski norm $|\cdot|$ which is $C^{\infty}$ on $\mathbb{R}^{n} \backslash\{0\}$ induces a Finsler structure in a natural way through the identification between $T_{x} \mathbb{R}^{n}$ and $\mathbb{R}^{n}$. Then $\left(\mathbb{R}^{n},|\cdot|, \operatorname{vol}_{n}\right)$ has the flat flag curvature. Hence a Banach or Minkowski space $\left(\mathbb{R}^{n},|\cdot|, \operatorname{vol}_{n}\right)$ satisfies $\mathrm{CD}(0, n)$ by Theorem 7.3 for $C^{\infty}$-norms, and by Theorem [5.6 via approximations for general norms.

(b) (Banach/Minkowski spaces with log-concave measures) A Banach or Minkowski space $\left(\mathbb{R}^{n},|\cdot|, m\right)$ equipped with a measure $m=e^{-\psi} \operatorname{vol}_{n}$ such that $\psi$ is $K$-convex with respect to $|\cdot|$ satisfies $\mathrm{CD}(K, \infty)$. Here the $K$-convexity means that

$$
\psi((1-t) x+t y) \leq(1-t) \psi(x)+t \psi(y)-\frac{K}{2}(1-t) t|x-y|^{2}
$$

holds for all $x, y \in \mathbb{R}^{n}$ and $t \in[0,1]$. This is equivalent to $\partial_{v}^{2} \psi \geq K$ (in the sense of (17.3)) if $|\cdot|$ and $\psi$ are $C^{\infty}$ (on $\mathbb{R}^{n} \backslash\{0\}$ and $\mathbb{R}^{n}$, respectively). Hence $\operatorname{CD}(K, \infty)$ again follows from Theorem 7.3 together with Theorem 5.6.

In particular, a Gaussian type space $\left(\mathbb{R}^{n},|\cdot|, e^{-|\cdot|^{2} / 2} \operatorname{vol}_{n}\right)$ satisfies $\operatorname{CD}(0, \infty)$ independently of $n$. It also satisfies $\operatorname{CD}(K, \infty)$ for some $K>0$ if (and only if) it is 2-uniformly convex in the sense that $|\cdot|^{2} / 2$ is $C^{-2}$-convex for some $C \geq 1$ (see [BCL] and [Oh4]), and then $K=C^{-2}$. For instance, $\ell_{p}$-spaces with $p \in(1,2]$ are 2-uniformly convex with $C=1 / \sqrt{p-1}$, and hence satisfies $\operatorname{CD}(p-1, \infty)$. Compare this with Example 4.5. 
(c) (Randers spaces) A Randers space $(M, F)$ is a special kind of Finsler manifold such that

$$
F(v)=\sqrt{g(v, v)}+\beta(v)
$$

for some Riemannian metric $g$ and a one-form $\beta$. We suppose that $|\beta(v)|^{2}<g(v, v)$ unless $v=0$, then $F$ is indeed a Finsler structure. Randers spaces are important in applications and reasonable for concrete calculations. In fact, we can see by calculation that $\mathbf{S}(v)=\partial_{v} \Psi \equiv 0$ holds if and only if $\beta$ is a Killing form of constant length as well as $m$ is the Busemann-Hausdorff measure (see [Oh7], [Sh2, Section 7.3] for more details). This means that there are many Finsler manifolds which do not admit any measures of $\operatorname{Ric}_{n} \geq K>-\infty$, and then we must consider $\operatorname{Ric}_{N}$ for $N>n$.

(d) (Hilbert geometry) Let $D \subset \mathbb{R}^{n}$ be a bounded open set with smooth boundary such that its closure $\bar{D}$ is strictly convex. Then the associated Hilbert distance is defined by

$$
d\left(x_{1}, x_{2}\right):=\log \left(\frac{\left\|x_{1}-x_{2}^{\prime}\right\| \cdot\left\|x_{2}-x_{1}^{\prime}\right\|}{\left\|x_{1}-x_{1}^{\prime}\right\| \cdot\left\|x_{2}-x_{2}^{\prime}\right\|}\right)
$$

for distinct $x_{1}, x_{2} \in D$, where $\|\cdot\|$ is the standard Euclidean norm and $x_{1}^{\prime}, x_{2}^{\prime}$ are intersections of $\partial D$ and the line passing through $x_{1}, x_{2}$ such that $x_{i}^{\prime}$ is on the side of $x_{i}$. Hilbert geometry is known to be realized by a Finsler structure with constant negative flag curvature. However, it is still unclear if it carries a (natural) measure for which the curvature-dimension condition holds.

(e) (Teichmüller space) Teichmüller metric on Teichmüller space is one of the most famous Finsler structures in differential geometry. It is known to be complete, while the Weil-Petersson metric is incomplete and Riemannian. The author does not know any investigation concerned with the curvature-dimension condition of Teichmüller space.

\subsection{Remarks and potential applications}

Due to celebrated work of Cheeger and Colding [CC], we know that a (non-Hilbert) Banach space can not be the limit space of a sequence of Riemannian manifolds (with respect to the measured Gromov-Hausdorff convergence) with a uniform lower Ricci curvature bound. Therefore the fact that Finsler manifolds satisfy the curvature-dimension condition means that it is too weak to characterize limit spaces of Riemannian manifolds. This should be compared with the following facts.

(I) A Banach space can be an Alexandrov space only if it happens to be a Hilbert space (and then it has the nonnegative curvature);

(II) It is not known if all Alexandrov spaces $X$ of curvature $\geq k$ can be approximated by a sequence of Riemannian manifolds $\left\{M_{i}\right\}_{i \in \mathbb{N}}$ of curvature $\geq k^{\prime}$.

We know that there are counterexamples to (II) if we impose the non-collapsing condition $\operatorname{dim} M_{i} \equiv \operatorname{dim} X$ (see $[\mathrm{Ka}]$ ), but the general situation admitting collapsing $\left(\operatorname{dim} M_{i}>\operatorname{dim} X\right)$ is still open and is one of the most important and challenging questions in Alexandrov geometry. Thus the curvature-dimension condition is not as good as the Alexandrov-Toponogov triangle comparison condition from the purely Riemannian geometric viewpoint. 
From a different viewpoint, Cheeger and Colding's observation means that the family of Finsler spaces is properly much wider than the family of Riemannian spaces. Therefore the validity of the curvature-dimension condition for Finsler manifolds opens the door to broader applications. Here we mention two of them.

(A) (The geometry of Banach spaces) Although their interested spaces are common to some extent, there is almost no connection between the geometry of Banach spaces and Finsler geometry (as far as the author knows). We believe that our differential geometric technique would be useful in the geometry of Banach spaces. For instance, Theorem 7.3 (together with Theorem [5.6) could recover and generalize Gromov and Milman's normal concentration of unit spheres in 2-uniformly convex Banach spaces (see [GM2] and [Le, Section 2.2]). To be precise, as an application of Theorem [7.3, we know the normal concentration of Finsler manifolds such that Ric $\infty$ goes to infinity (see [Oh5], and [GM1], [Le, Section 2.2] for the Riemannian case). This seems to imply the concentration of unit spheres mentioned above.

(B) (Approximations of graphs) Generally speaking, Finsler spaces give much better approximations of graphs than Riemannian spaces, when we impose a lower Ricci curvature bound. For instance, Riemannian spaces into which the $\mathbb{Z}^{n}$-lattice is nearly isometrically embedded should have very negative curvature, while the $\mathbb{Z}^{n}$-lattice is isometrically embedded in flat $\ell_{1}^{n}$. This kind of technique seems useful for investigating graphs with Ricci curvature bounded below (in some sense), and provides a different point of view on variants of the curvature-dimension condition for discrete spaces (see, e.g., [Ol], [BoS]).

Further Reading We refer to [BCS] and [Sh2] for the fundamentals of Finsler geometry and important examples. The interpretation of the flag curvature using vector fields can be found in [Sh2, Chapter 6]. We also refer to [Sh1] and [Sh3 for the S-curvature and its applications including a volume comparison theorem different from Theorem 6.3 (which has some topological applications). The S-curvature of Randers spaces and the characterization of its vanishing (Example 7.4(c)) are studied in [Sh2, Section 7.3] and Oh7].

Definition 7.2 and Theorem 7.3 are due to Oh5], while the weight function $\Psi$ on $S M$ has already been considered in the definition of $\mathbf{S}$-curvature. See also [OhS] for related work concerning heat flow on Finsler manifolds, and [Oh6] for a survey on these subjects. The curvature-dimension condition $\operatorname{CD}(0, n)$ of Banach spaces (Example 7.4(a)) is first demonstrated by Cordero-Erausquin (see [Vi2, page 908]).

\section{Related topics}

We briefly comment on further related topics.

\subsection{Non-branching spaces}

We say that a geodesic space $(X, d)$ is non-branching if geodesics do not branch in the sense that each quadruple of points $z, x_{0}, x_{1}, x_{2} \in X$ with $d\left(x_{0}, x_{1}\right)=d\left(x_{0}, x_{2}\right)=2 d\left(z, x_{i}\right)$ $(i=0,1,2)$ must satisfy $x_{1}=x_{2}$. This is a quite useful property. For instance, in such a space satisfying $\mathrm{CD}(K, N)$ for some $K$ and $N$, a.e. $x \in X$ has unique minimal geodesic 
from $x$ to a.e. $y \in X$ ([St4, Lemma 4.1]). Therefore we can localize the inequality (5.3), and then (5.3) for single $U=S_{N}$ implies that for all $U \in \mathcal{D C}_{N}$ (see Remark 5.2(e), (f), [St4, Proposition 4.2]). There are some more results known only in non-branching spaces (see, e.g., [St4, Section 4], [FV] and also Subsection 8.3 below).

Riemannian (or Finsler) manifolds and Alexandrov spaces are clearly non-branching. However, as $n$-dimensional Banach and Minkowski spaces satisfy $\operatorname{CD}(0, n)$, the curvaturedimension condition does not prevent the branching phenomenon. One big open problem after Cheeger and Colding's work $[\mathrm{CC}]$ is whether any limit space of Riemannian manifolds with a uniform lower Ricci curvature bound is non-branching or not.

\subsection{Alexandrov spaces}

As was mentioned in Remark 3.1. Alexandrov spaces are metric spaces whose sectional curvature is bounded from below in terms of the triangle comparison property (see [BGP], [OtS], [BBI, Chapters 4, 10] for more details). One interesting fact is that a compact geodesic space $(X, d)$ is an Alexandrov space of nonnegative curvature if and only if so is the Wasserstein space $\left(\mathcal{P}(X), d_{2}^{W}\right)$ over it ([St3, Proposition 2.10], [LV2, Theorem A.8]). This is a metric geometric explanation of Otto's formal calculation of the sectional curvature of $\left(\mathcal{P}_{2}\left(\mathbb{R}^{n}\right), d_{2}^{W}\right)([\mathrm{Ot}])$. We remark that this relation can not be extended to positive or negative curvature bounds. In fact, if $(X, d)$ is not an Alexandrov space of nonnegative curvature, then $\left(\mathcal{P}(X), d_{2}^{W}\right)$ is not an Alexandrov space of curvature $\geq k$ even for negative $k$ ([St3, Proposition 2.10]). Optimal transport in Alexandrov spaces is further studied in [Be], Oh3], SSav, Gi] and GO].

Since the Ricci curvature is the trace of the sectional curvature, it is natural to expect that Alexandrov spaces satisfy the curvature-dimension condition. Petrunin [Pe3] recently claims that it is indeed the case for $K=0$, and is extended to the general case $K \neq 0$ by [ZZ]. They use the second variation formula in [Pe1] and the gradient flow technique developed in [PP] and [Pe2], instead of calculations as in Sections [3, 4 involving Jacobi fields.

\subsection{The measure contraction property}

For $K \in \mathbb{R}$ and $N \in(1, \infty)$, a metric measure space is said to satisfy the measure contraction property $\mathrm{MCP}(K, N)$ if the Bishop inequality (3.3) holds in an appropriate sense. More precisely, $\operatorname{MCP}(K, N)$ for $(X, d, m)$ means that any $x \in X$ admits a measurable $\operatorname{map} \Phi: X \longrightarrow \Gamma(X)$ satisfying $e_{0} \circ \Phi \equiv x, e_{1} \circ \Phi=\operatorname{Id}_{X}$ and

$$
d m \geq\left(e_{t} \circ \Phi\right)_{\sharp}\left(t^{N} \beta_{K, N}^{t}(d(x, y)) d m(y)\right)
$$

for all $t \in(0,1)$ as measures (compare this with Theorem 6.1(i)). As we mentioned in Further Reading in Section 3, this kind of property was suggested in [CC, I, Appendix 2] and [Gr, Section 5.I], and systematically studied in [Oh1], Oh2] and [St4, Sections 5, 6]. Some variants have been also studied in [KS1] and [St1] before them.

$\operatorname{MCP}(K, N)$ can be regarded as the curvature-dimension condition $\operatorname{CD}(K, N)$ applied only for each pair of a Dirac measure and a uniform distribution on a set, and CD actually 
implies MCP in non-branching spaces. It is known that Alexandrov spaces satisfy MCP (see [Oh1, KS2]). For $n$-dimensional (unweighted) Riemannian manifolds, $\operatorname{MCP}(K, n)$ is equivalent to Ric $\geq K$, however, $\operatorname{MCP}(K, N)$ with $N>n$ does not imply Ric $\geq K$. In fact, a sufficiently small ball in $\mathbb{R}^{n}$ equipped with the Lebesgue measure satisfies $\operatorname{MCP}(1, n+1)$. This is one drawback of MCP. On the other hand, an advantage of MCP is its simpleness, there are several facts known for MCP and unknown for CD. We shall compare these properties in more details.

(A) (Product spaces $\left(L^{2}\right.$-tensorization property)) If $\left(X_{i}, d_{i}, m_{i}\right)$ satisfies $\operatorname{MCP}\left(K_{i}, N_{i}\right)$ for $i=1,2$, then the product metric measure space $\left(X_{1} \times X_{2}, d_{1} \times d_{2}, m_{1} \times m_{2}\right)$ satisfies $\operatorname{MCP}\left(\min \left\{K_{1}, K_{2}\right\}, N_{1}+N_{2}\right)$ ([Oh2]). The analogous property for CD is known only for $\min \left\{K_{1}, K_{2}\right\}=0$ or $N_{1}+N_{2}=\infty$ in non-branching spaces ([St3]).

Recently, Bacher and Sturm [BaS1] introduce a slightly weaker variant of CD, called the reduced curvature-dimension condition CD* (recall (4.15) in Further Reading of Section 4). They show that $\mathrm{CD}^{*}$ enjoys the tensorization property if the spaces in consideration are non-branching.

(B) (Euclidean cones) If $(X, d, m)$ satisfies $\operatorname{MCP}(N-1, N)$, then its Euclidean cone $\left(C X, d_{C X}, m_{C X}\right)$ defined by

$$
\begin{aligned}
C X & :=(X \times[0, \infty)) / \sim, \quad(x, 0) \sim(y, 0), \\
d_{C X}((x, s),(y, t)) & :=\sqrt{s^{2}+t^{2}-2 s t \cos d(x, y)}, \\
d m_{C X} & :=d m \times\left(t^{N} d t\right)
\end{aligned}
$$

satisfies $\operatorname{MCP}(0, N+1)([\mathrm{Oh} 2])$. This is recently established for the curvature-dimension condition by [BaS2] in the case where $(X, d, m)$ is Riemannian.

(C) (Local-to-global property) Sturm [St3] shows that, if $(X, d, m)$ is non-branching and if every point in $X$ admits an open neighborhood on which $\operatorname{CD}(K, \infty)$ holds, then the whole space $(X, d, m)$ globally satisfies $\operatorname{CD}(K, \infty)$. In other words, $\operatorname{CD}(K, \infty)$ is a local condition as is the Ricci curvature bound on a Riemannian manifold. The same holds true also for $\mathrm{CD}(0, N)$ with $N<\infty$. It is shown in [BaS1] that $\mathrm{CD}^{*}(K, N)$ satisfies the local-to-global property for general $K \in \mathbb{R}$ and $N \in(1, \infty)$, however, it is still open and unclear if $\operatorname{CD}(K, N)$ for $K \neq 0$ and $N<\infty$ is a local condition.

In contrast, the local-to-global property is known to be false for MCP. As we mentioned above, sufficiently small balls in $\mathbb{R}^{n}$ satisfy $\operatorname{MCP}(1, n+1)$, while the entire space $\mathbb{R}^{n}$ does not satisfy it by virtue of the Bonnet-Myers diameter bound (Theorem 6.5).

\section{References}

[AGS] L. Ambrosio, N. Gigli and G. Savaré, Gradient flows in metric spaces and in the space of probability measures, Birkhäuser Verlag, Basel, 2005.

[BaS1] K. Bacher and K.-T. Sturm, Localization and tensorization properties of the curvature-dimension condition for metric measure spaces, J. Funct. Anal. 259 (2010), 28-56.

[BaS2] K. Bacher and K.-T. Sturm, Ricci bounds for Euclidean and spherical cones, Preprint (2010). Available at arXiv:1003.2114 
[BE] D. Bakry and M. Émery, Diffusions hypercontractives (French), Séminaire de probabilités, XIX, 1983/84, 177-206, Lecture Notes in Math. 1123, Springer, Berlin, 1985.

[BCL] K. Ball, E. A. Carlen and E. H. Lieb, Sharp uniform convexity and smoothness inequalities for trace norms, Invent. Math. 115 (1994), 463-482.

[Bal] W. Ballmann, Lectures on spaces of nonpositive curvature. With an appendix by Misha Brin, Birkhäuser Verlag, Basel, 1995.

[BCS] D. Bao, S.-S. Chern and Z. Shen, An introduction to Riemann-Finsler geometry, Springer-Verlag, New York, 2000.

[Bay] V. Bayle, Propriétés de concavité du profil isopérimétrique et applications (French), Thèse de Doctorat, Institut Fourier, Université Joseph-Fourier, Grenoble, 2003.

[Be] J. Bertrand, Existence and uniqueness of optimal maps on Alexandrov spaces, Adv. Math. 219 (2008), 838-851.

[BoS] A.-I. Bonciocat and K.-T. Sturm, Mass transportation and rough curvature bounds for discrete spaces, J. Funct. Anal. 256 (2009), 2944-2966.

[Br] Y. Brenier, Polar factorization and monotone rearrangement of vector-valued functions, Comm. Pure Appl. Math. 44 (1991), 375-417.

[BBI] D. Burago, Yu. Burago and S. Ivanov, A course in metric geometry, American Mathematical Society, Providence, RI, 2001.

[BGP] Yu. Burago, M. Gromov and G. Perel'man, A. D. Alexandrov spaces with curvatures bounded below, Russian Math. Surveys 47 (1992), 1-58.

[Ch] I. Chavel, Riemannian geometry. A modern introduction. Second edition, Cambridge University Press, Cambridge, 2006.

[CC] J. Cheeger and T. H. Colding, On the structure of spaces with Ricci curvature bounded below. I, II, III, J. Differential Geom. 46 (1997), 406-480; ibid. 54 (2000), 13-35; ibid. 54 (2000), 37-74.

[CE] J. Cheeger and D. G. Ebin, Comparison theorems in Riemannian geometry, Revised reprint of the 1975 original, AMS Chelsea Publishing, Providence, RI, 2008.

[CG] J. Cheeger and D. Gromoll, The splitting theorem for manifolds of nonnegative Ricci curvature, J. Differential Geometry 6 (1971/72), 119-128.

[CMS1] D. Cordero-Erausquin, R. J. McCann and M. Schmuckenschläger, A Riemannian interpolation inequality à la Borell, Brascamp and Lieb, Invent. Math. 146 (2001), 219-257. 
[CMS2] D. Cordero-Erausquin, R. J. McCann and M. Schmuckenschläger, PrékopaLeindler type inequalities on Riemannian manifolds, Jacobi fields, and optimal transport, Ann. Fac. Sci. Toulouse Math. (6) 15 (2006), 613-635.

[FF] A. Fathi and A. Figalli, Optimal transportation on non-compact manifolds, Israel J. Math. 175 (2010), 1-59.

[FG] A. Figalli and N. Gigli, Local semiconvexity of Kantorovich potentials on noncompact manifolds, To appear in ESAIM Control Optim. Calc. Var.

[FV] A. Figalli and C. Villani, Strong displacement convexity on Riemannian manifolds, Math. Z. 257 (2007), 251-259.

[Fu] K. Fukaya, Collapsing of Riemannian manifolds and eigenvalues of Laplace operator, Invent. Math. 87 (1987), 517-547.

[Ga] R. J. Gardner, The Brunn-Minkowski inequality, Bull. Amer. Math. Soc. (N.S.) 39 (2002), 355-405.

[Gi] N. Gigli, On the inverse implication of Brenier-McCann theorems and the structure of $\left(\mathscr{P}_{2}(M), W_{2}\right)$, Preprint (2009). Available at http://cvgmt.sns.it/people/gigli/

[GO] N. Gigli and S. Ohta, First variation formula in Wasserstein spaces over compact Alexandrov spaces, Preprint (2010). Available at http://www.math.kyoto-u.ac.jp/ sohta/

[Gr] M. Gromov, Metric structures for Riemannian and non-Riemannian spaces, Birkhäuser, Boston, MA, 1999.

[GM1] M. Gromov and V. D. Milman, A topological application of the isoperimetric inequality, Amer. J. Math. 105 (1983), 843-854.

[GM2] M. Gromov and V. D. Milman, Generalization of the spherical isoperimetric inequality to uniformly convex Banach spaces, Compositio Math. 62 (1987), 263282.

[Ka] V. Kapovitch, Regularity of limits of noncollapsing sequences of manifolds, Geom. Funct. Anal. 12 (2002), 121-137.

[KS1] K. Kuwae and T. Shioya, On generalized measure contraction property and energy functionals over Lipschitz maps, ICPA98 (Hammamet). Potential Anal. 15 (2001), 105-121.

[KS2] K. Kuwae and T. Shioya, Infinitesimal Bishop-Gromov condition for Alexandrov spaces, Adv. Stud. Pure Math. 57 (2010), 293-302.

[KS3] K. Kuwae and T. Shioya, A topological splitting theorem for weighted Alexandrov spaces, Preprint (2009). Available at arXiv:0903.5150 
[Le] M. Ledoux, The concentration of measure phenomenon, American Mathematical Society, Providence, RI, 2001.

[Lo1] J. Lott, Some geometric properties of the Bakry-Émery-Ricci tensor, Comment. Math. Helv. 78 (2003), 865-883.

[Lo2] J. Lott, Optimal transport and Ricci curvature for metric-measure spaces, Surveys in differential geometry XI, 229-257, Int. Press, Somerville, MA, 2007.

[LV1] J. Lott and C. Villani, Weak curvature conditions and functional inequalities, J. Funct. Anal. 245 (2007), 311-333.

[LV2] J. Lott and C. Villani, Ricci curvature for metric-measure spaces via optimal transport, Ann. of Math. 169 (2009), 903-991.

[Mc1] R. J. McCann, A convexity principle for interacting gases, Adv. Math. 128 (1997), 153-179.

[Mc2] R. J. McCann, Polar factorization of maps on Riemannian manifolds, Geom. Funct. Anal. 11 (2001), 589-608.

[Mo] F. Morgan, Geometric measure theory. A beginner's guide. Fourth edition, Elsevier/Academic Press, Amsterdam, 2009.

[Oh1] S. Ohta, On the measure contraction property of metric measure spaces, Comment. Math. Helv. 82 (2007), 805-828.

[Oh2] S. Ohta, Products, cones, and suspensions of spaces with the measure contraction property, J. Lond. Math. Soc. (2) 76 (2007), 225-236.

[Oh3] S. Ohta, Gradient flows on Wasserstein spaces over compact Alexandrov spaces, Amer. J. Math. 131 (2009), 475-516.

[Oh4] S. Ohta, Uniform convexity and smoothness, and their applications in Finsler geometry, Math. Ann. 343 (2009), 669-699.

[Oh5] S. Ohta, Finsler interpolation inequalities, Calc. Var. Partial Differential Equations 36 (2009), 211-249.

[Oh6] S. Ohta, Optimal transport and Ricci curvature in Finsler geometry, Adv. Stud. Pure Math. 57 (2010), 323-342.

[Oh7] S. Ohta, Vanishing S-curvature of Randers spaces, Preprint (2009). Available at arXiv:0909.1399

[OhS] S. Ohta and K.-T. Sturm, Heat flow on Finsler manifolds, Comm. Pure Appl. Math. 62 (2009), 1386-1433.

[OT] S. Ohta and A. Takatsu, Displacement convexity of generalized entropies, Preprint (2010). Available at arXiv:1005:1331 
[Ol] Y. Ollivier, Ricci curvature of Markov chains on metric spaces, J. Funct. Anal. 256 (2009), 810-864.

[OtS] Y. Otsu, T. Shioya, The Riemannian structure of Alexandrov spaces, J. Differential Geom. 39 (1994), 629-658.

[Ot] F. Otto, The geometry of dissipative evolution equations: the porous medium equation, Comm. Partial Differential Equations 26 (2001), 101-174.

[OV] F. Otto and C. Villani, Generalization of an inequality by Talagrand and links with the logarithmic Sobolev inequality, J. Funct. Anal. 173 (2000), 361-400.

[PP] G. Perel'man and A. Petrunin, Quasigeodesics and gradient curves in Alexandrov spaces, Unpublished preprint (1994).

[Pe1] A. Petrunin, Parallel transportation for Alexandrov space with curvature bounded below, Geom. Funct. Anal. 8 (1998), 123-148.

[Pe2] A. Petrunin, Semiconcave functions in Alexandrov's geometry, Surveys in differential geometry. Vol. XI, 137-201, Surv. Differ. Geom. 11, Int. Press, Somerville, MA, 2007.

[Pe3] A. Petrunin, Alexandrov meets Lott-Villani-Sturm, Preprint (2009). Available at arXiv:1003.5948

[Qi] Z. Qian, Estimates for weighted volumes and applications, Quart. J. Math. Oxford Ser. (2) 48 (1997), 235-242.

[vRS] M.-K. von Renesse and K.-T. Sturm, Transport inequalities, gradient estimates, entropy and Ricci curvature, Comm. Pure Appl. Math. 58 (2005), 923-940.

[Sak] T. Sakai, Riemannian geometry, Translated from the 1992 Japanese original by the author. Translations of Mathematical Monographs, 149. American Mathematical Society, Providence, RI, 1996.

[Sav] G. Savaré, Gradient flows and diffusion semigroups in metric spaces under lower curvature bounds, C. R. Math. Acad. Sci. Paris 345 (2007), 151-154.

[Sh1] Z. Shen, Volume comparison and its applications in Riemann-Finsler geometry, Adv. Math. 128 (1997), 306-328.

[Sh2] Z. Shen, Lectures on Finsler geometry, World Scientific Publishing Co., Singapore, 2001.

[Sh3] Z. Shen, Landsberg curvature, S-curvature and Riemann curvature, A sampler of Riemann-Finsler geometry, 303-355, Math. Sci. Res. Inst. Publ., 50, Cambridge Univ. Press, Cambridge, 2004.

[St1] K.-T. Sturm, Diffusion processes and heat kernels on metric spaces, Ann. Probab. 26 (1998), 1-55. 
[St2] K.-T. Sturm, Convex functionals of probability measures and nonlinear diffusions on manifolds, J. Math. Pures Appl. 84 (2005), 149-168.

[St3] K.-T. Sturm, On the geometry of metric measure spaces. I, Acta Math. 196 (2006), 65-131.

[St4] K.-T. Sturm, On the geometry of metric measure spaces. II, Acta Math. 196 (2006), 133-177.

[Vi1] C. Villani, Topics in optimal transportation, American Mathematical Society, Providence, RI, 2003.

[Vi2] C. Villani, Optimal transport, old and new, Springer-Verlag, Berlin, 2009.

[We] G. Wei, Manifolds with a lower Ricci curvature bound, Surveys in differential geometry XI, 203-227, Int. Press, Somerville, MA, 2007.

[ZZ] H.-C. Zhang and X.-P. Zhu, Ricci curvature on Alexandrov spaces and rigidity theorems, Preprint (2009). Available at arXiv:0912.3190 